\title{
Control of T-S Fuzzy Systems Using Fuzzy Weighting-Dependent Lyapunov Function
}

\author{
Sung Hyun Kim and PooGyeon Park \\ Division of Electrical and Computer Engineering, Pohang University of Science \\ and Technology, Pohang, Kyungbuk, 790-784,
}

Korea

\section{Introduction}

Over the past two decades, there has been a rapidly growing interest in approximating a nonlinear system by a Takagi-Sugeno (T-S) fuzzy model (Takagi \& Sugeno, 1985). In general, this model is represented by using a set of fuzzy rules to describe a global nonlinear system in terms of a set of local linear models which are smoothly connected by fuzzy membership functions. Based on the T-S fuzzy model, recently, various fuzzy controllers have been developed under the so-called parallel-distributed compensation (PDC) scheme (in which each control rule is distributively designed for the corresponding rule of a T-S fuzzy model) and have been widely and successfully applied in fields ranging from aerospace to process control. The reason is because the fuzzy model-based control method provides a natural, simple and effective design approach to complement other nonlinear control techniques that require special and rather involved knowledge. Below are listed the main features of T-S model-based fuzzy control method:

1. It does not require severe structural assumptions on the plant model.

2. It preserves well-understood linear intuition.

3. It is naturally compatible with decompositions of the overall control problem. The decompositions are typically not hierarchical, and the interactions of sub-problems are captured by physical variables that are typically state variables in a more complete model of the overall system.

4. It enables control systems to respond rapidly to changing operating conditions. For this reason, it is important that the selected physical variables reflect changes in plant dynamics as operating conditions change.

In fact, the T-S model-based fuzzy control method (of divide and conquer type) constructs a nonlinear controller, with certain required dynamic properties, by combining, in some sense, the members of appropriate family of linear time-invariant controllers. Here, nonlinear control design task is broken into a number of linear sub-problems, which enable linear design methods to be applied to nonlinear problems. Within the general framework of the T-S fuzzy model-based control method, a flurry of research activities have quickly yielded many important results on the design of fuzzy control systems by means of the following Lyapunov function approaches:

1. Common quadratic Lyapunov function approach (Tanaka \& Sugeno, 1992; Tanaka et al, 1996; Wang et al, 1996; Cao \& Frank, 2000; Assawinchaichote, 2004).

Source: Fuzzy Systems, Book edited by: Ahmad Taher Azar,

ISBN 978-953-7619-92-3, pp. 216, February 2010, INTECH, Croatia, downloaded from SCIYO.COM 
2. Piecewise Lyapunov function approach (Cao et al, 1996; Cao et al, 1997; Cao et al, 1999; Han et al, 2000; Feng, 2003; Feng, 2004; Chen et al, 2005).

3. Fuzzy weighting-dependent Lyapunov function approach (Tanaka et al, 2001; Park \& Choi, 2001; Choi \& Park, 2003; Kim \& Park 2008; Kim et al, 2009).

The basic idea of these approaches is to design a feedback controller for each local model and to construct a global controller from the local controller in such a way that global stability of the closed-loop fuzzy control system is guaranteed. In the context of these approaches, various studies have attempted to tackle the robust control problem (Tanaka et al, 1996; Chen et al, 1999; Tsai \& Li, 2009), performance-oriented control problem (Chen et al, 2000; Xiaodong \& Qingling, 2003; Zhou et al, 2005), networked control problem (Hwang \& Chang, 2008; Jiang \& Han, 2008; Gao et al, 2009), and delayed system control problem (Cao \& Frank, 2000; Chen et al, 2005; Wu, 2008).

\section{A. T-S fuzzy model and control synthesis}

In general, it is not possible to exactly reformulate a nonlinear system as a T-S fuzzy system. However, it is possible to over-bound the nonlinear system in the sense that every solution to the nonlinear system is a solution to the T-S fuzzy system (but not vice versa). Thus, rather conservative results are expected in the procedure of modeling the T-S fuzzy system. Moreover, since the T-S fuzzy model is not unique, there may be a potential in reducing the conservatism occurring when approximating the nonlinear system. Hence, one always needs to discuss how to non-conservatively construct the T-S fuzzy model for the given nonlinear system. As a result, various methods for the reformulation of nonlinear systems into T-S fuzzy systems have been presented in the literature (Tanaka \& Wang, 2001; and references therein). In this chapter, we would like to introduce a geometric method for some particular nonlinearities: sector nonlinearity, saturation nonlinearity, and fault nonlinearity (see Section 2).

Meanwhile, based on the aforementioned Lyapunov function approaches, numerous investigations and researches have been carried out to develop the T-S model-based fuzzy control system. Here, it should be noted that recent research efforts have focused on using the PLF or FWLF approach when establishing a feedback control law since the CQLF approach leads to over-conservative design solutions for a large number of T-S fuzzy subsystems. Thus, we shall also focus on taking advantage of the FWLF approach to derive less conservative conditions for the solvability of the stabilization problem (for lack of space, the PLF approach will be not discussed in this chapter).

\section{B. Main issues}

Most stabilization conditions based on the FWLF are formulated in terms of parameterized linear matrix inequalities (PLMIs), which causes the following primary practical difficulty: the PLMI-based condition involves an infinite number of LMI-based conditions and thus the task of establishing a controller is intractable numerically. This arises because the PLMIbased condition must be satisfied for every allowable parameter value that leads to uncountably many conditions since there is a continuum of parameter values. To overcome this difficulty, Becker et al (1993) proposed an approximate, ad hoc approach whereby the parameter space is divided into a fine grid, and a controller is designed so that the solvability conditions are satisfied at a finite number of parameter values. However, it should be noted that there appears to be little guidance as to how perform the gridding. 
Moreover, for a particular grid spacing, the number of grid points required grows extremely rapidly as the number of parameters increases. Hence, despite the relative efficiency of the available numerical algorithms for solving linear matrix inequalities (LMIs), the utility of this approach with present computing facilities is strictly limited to systems with a small number of parameters (less than three or four).

To deal with this problem, we shall select an appropriate structure for variables, say $\mathcal{X}(\theta(t))$, of the PLMI-based condition under consideration in such a way that the variables are polynomially dependent on parameters denoting fuzzy weighting functions, say $\theta_{i}(t)$ :

$$
\mathcal{X}(\theta(t))=\sum_{i_{1}=1}^{r} \sum_{i_{2}=1}^{r} \cdots \sum_{i_{g}=1}^{r} \theta_{i_{1}}(t) \theta_{i_{2}}(t) \cdots \theta_{i_{g}}(t) \mathcal{X}_{i_{1} i_{2} \cdots i_{g}}
$$

subject to $\sum_{i=1}^{r} \theta_{i}(t)=1$ and $\theta_{i}(t) \geq 0, i=1, \cdots, r$. In particular, for simplicity of presentation, we shall take the following two cases into consideration in this chapter:

Affine Parameter Dependence (APD):

$$
\mathcal{X}(\theta(t))=\sum_{i=1}^{r} \theta_{i}(t) \mathcal{X}_{i}, \text { s.t. } \sum_{i=1}^{r} \theta_{i}(t)=1, \theta_{i}(t) \geq 0, i=1, \cdots, r,
$$

Quadratic Parameter Dependence (QPD):

$$
\mathcal{X}(\theta(t))=\sum_{i=1}^{r} \sum_{j=1}^{r} \theta_{i}(t) \theta_{j}(t) \mathcal{X}_{i j}, \text { s.t. } \sum_{i=1}^{r} \theta_{i}(t)=1, \theta_{i}(t) \geq 0, i=1, \cdots, r,
$$

In fact, the use of (1) yields a polynomially parameter-dependent condition such as

$$
0<\sum_{i_{1}=1}^{r} \sum_{i_{2}=1}^{r} \cdots \sum_{i_{p}=1}^{r} \theta_{i_{1}}(t) \theta_{i_{2}}(t) \cdots \theta_{i_{p}}(t) \mathcal{L}_{i_{1} i_{2} \cdots i_{p}}
$$

subject to $\sum_{i=1}^{r} \theta_{i}(t)=1$ and $\theta_{i}(t) \geq 0, i=1, \cdots, r$. Thus, the condition in (4) naturally reduces to a feasibility problem with a finite number of conditions as follows:

$$
0<\mathcal{L}_{i_{1} i_{2} \cdots i_{p}}, \forall i_{1}, i_{2}, \cdots, i_{p} \in[1, r]
$$

However, it is very conservative to use (5) to numerically solve the feasible problem of (4): Thus, we shall propose an efficient relaxation technique to reduce the conservatism caused by the use of (5) (see Section 3), which may achieve better system performances compared with those of other techniques appeared in the literature (Tanaka et al, 1998; Kim \& Lee, 2000; Xiaodong \& Qingling, 2003; Tuan et al, 2001; Teixeira, 2003; Sala \& Ariño , 2007; Fang et al, 2006).

\section{Organization}

This chapter is organized as follows: Section 2 gives the information on the T-S fuzzy system description and its modeling. Further, Section 3 illustrates about the parameterized linear 
matrix inequality (PLMI) and introduces our main relaxation technique in detail. Based on CQLFs and FWLFs, Section 4 gives the LMI-based stabilization conditions, derived using the proposed relaxation technique in Section 3, for a class of T-S fuzzy systems.

Notation and symbols

We collect here, for ease of reference, a list of the main notation and symbols that represent the same meaning throughout the chapters.

$\mathcal{R}^{n} \quad$ denotes the $\mathrm{n}$-dimensional real space.

$\|x\|=\left(x^{T} x\right)^{1 / 2}$ is taken to be the standard Euclidian norm.

$\mathcal{L}_{2+}=\mathcal{L}_{2}[0, \infty)$ denotes the Lebesgue space consisting of square-integrable functions on $[0, \infty)$.

$\operatorname{diag}(A, B) \quad$ denotes a diagonal matrix with diagonal entries $A$ and $B$.

$A \oplus B \quad$ stands for the Kronecker sum of two matrices $A$ and $B$, which is the same as $\operatorname{diag}(A, B)$

(*) is used, in symmetric block matrices, as an ellipsis for terms that are induced by symmetry.

$X \geq Y \quad$ mean that $X-Y$ is positive semi-definite, respectively.

$X>Y \quad$ mean that $X-Y$ is positive definite, respectively.

$\operatorname{Tr}(Q) \quad$ returns the sum of the diagonal elements of the matrix $Q$.

\section{T-S fuzzy system description and modeling}

T-S fuzzy systems have recently received much attention in the engineering field, such as chemical processes, robotics systems, automatic systems, aerospace or vehicle systems, and manufacturing processes, owing to their ability to represent the nonlinear system and their systematic means of computing feedback controllers.

\section{A. T-S fuzzy system description}

The $i$ th rules of the T-S fuzzy models are of the following forms:

Model Rule $i:$ IF $\eta_{1}(t)$ is $\mathcal{F}_{i 1}$ and $\ldots$ and $\eta_{s}(t)$ is $\mathcal{F}_{i s}$, THEN

$$
\left\{\begin{array}{rl}
\nabla x(t) & =A_{i} x(t)+B_{i} u(t) \\
y(t) & =C_{i} x(t)
\end{array}, \text { for } i=1,2, \cdots, r,\right.
$$

where the consequent subsystems in (6) represent linear systems in local operating regions; $\mathcal{F}_{i j}$ denotes a fuzzy set; $\eta_{1}(t), \cdots, \eta_{s}(t)$ denote the premise variables of the model; $r$ denotes the number of IF - THEN rules; $x(t) \in \mathcal{R}^{n_{x}}, u(t) \in \mathcal{R}^{n_{u}}, y(t) \in \mathcal{R}^{n_{y}}$ denote the state, the input, the measured output, respectively; and $\nabla$ represents the derivative operator for continuous-time and the forward operator for discrete-time systems. Here, it is assumed that the premise variables not not explicitly depend on the control input $u(t)$. This assumption is needed to avoid a complicated defuzzification process of fuzzy controller, under which the overall fuzzy model is inferred as

$$
\left\{\begin{aligned}
\nabla x(t) & =A(\theta(t)) x(t)+B(\theta(t)) u(t) \\
y(t) & =C(\theta(t)) x(t)
\end{aligned}\right.
$$


where

$$
\begin{gathered}
{\left[\begin{array}{cc}
A(\theta(t)) & B(\theta(t)) \\
C(\theta(t)) & 0
\end{array}\right] \stackrel{\Delta}{=} \sum_{i=1}^{r} \theta_{i}(t)\left[\begin{array}{cc}
A_{i} & B_{i} \\
C_{i} & 0
\end{array}\right],} \\
\theta_{i}(t)=\theta_{i}(\eta(t))=\frac{g_{i}(\eta(t))}{\sum_{i=1}^{r} g_{i}(\eta(t))}, g_{i}(\eta(t))=\prod_{j=1}^{s} f_{i j}\left(\eta_{j}(t)\right)
\end{gathered}
$$

in which $A_{i}, B_{i}$, and $C_{i}$ are real constant matrices with appropriate dimensions, the notation $\eta(t)=\left[\eta_{1}(t), \cdots, \eta_{s}(t)\right]^{T} \in \mathcal{R}^{s}$, and $f_{i j}\left(\eta_{j}(t)\right)$ denotes the grade of membership of $\eta_{j}(t)$ in $\mathcal{F}_{i j}$, and $\theta(t)=\left[\theta_{1}(t), \cdots, \theta_{r}(t)\right] \in \mathcal{R}^{r}$. Moreover, let $g_{i}(\eta(t)) \geq 0$, for $i=1, \cdots, r$, and $\sum_{i=1}^{r} g_{i}(\eta(t))>0$ for all time $t$. Then, we can claim that $\theta_{i}(\eta(t)) \geq 0$, for $i=1, \cdots, r$, and $\sum_{i=1}^{r} \theta_{i}(\eta(t))=1$ for all time $t$.

As shown in (8), the T-S fuzzy system is defined as linear systems whose dynamics depend on time-varying parameters $\theta_{i}(t)$ referred to as the scheduling or weight sequence. Further, it is worth pointing out that the parameters $\theta_{i}(t)$ are generally subject to the following constraints:

\section{Continuous-Time Case:}

$$
\begin{gathered}
\bar{\theta}_{\min } \leq \bar{\theta}(t)\left(\stackrel{\Delta}{=} \sum_{i=1}^{r} \theta_{i}(t)\right) \leq \bar{\theta}_{\max } \\
\alpha_{i} \leq \theta_{i}(t) \leq \beta_{i}, \text { for } i=1, \cdots, r \\
\mu_{i} \leq \dot{\theta}_{i}(t) \leq v_{i}, \text { for } i=1, \cdots, r .
\end{gathered}
$$

Discrete-Time Case:

$$
\begin{gathered}
\bar{\theta}_{\min } \leq \bar{\theta}(t)\left(\stackrel{\Delta}{=} \sum_{i=1}^{r} \theta_{i}(t)\right) \leq \bar{\theta}_{\max }, \\
\alpha_{i} \leq \theta_{i} \leq \beta_{i}, \text { for } i=1, \cdots, r, \\
\left|\theta_{i}(t)-\theta_{i}(t-1)\right| \leq \delta_{i}, \text { for } i=1, \cdots, r .
\end{gathered}
$$

Remark 1 In general, the parameter $\theta_{i}(t)$ is a function of time $t$, states $x(t)$, and inputs $u(t)$. Except for the actuator nonlinearity (see Section 2-B), the parameter $\theta_{i}(t)$ is mostly associated with the state of the system in (7).

\section{B. T-S fuzzy model construction}

Nonlinear dynamic models for mechanical systems can be readily obtain by, for example, the Lagrange method and the Newton-Euler method. In such cases, we can represent the 

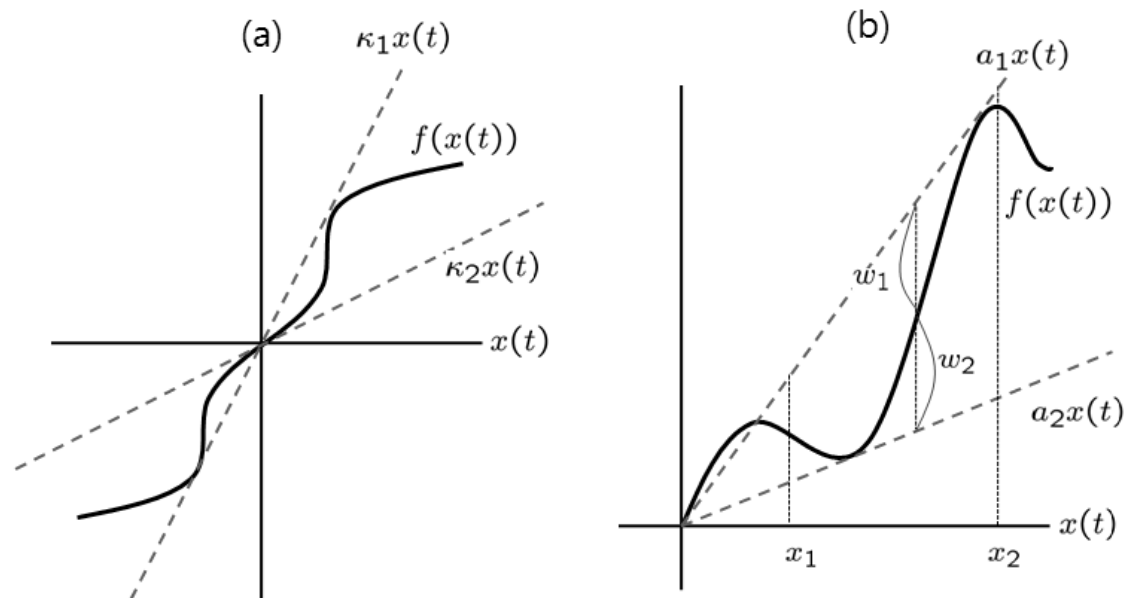

Fig. 1. (a) Global sector nonlinearity; and (b) local sector nonlinearity.

given nonlinear dynamical models as T-S fuzzy systems by using the idea of "sector nonlinearity", "saturation nonlinearity", or a combination of them. Prior to modeling an T-S fuzzy system, we need to simplify the original nonlinear model as much as possible. The procedure is important for practical applications since it always leads to the reduction of the number of the parameters $\theta_{i}(t)$, which plays an important role in reducing the effort for analysis and design of control systems.

\section{Sector Nonlinearity:}

Consider a simple nonlinear system $\nabla x(t)=f(x(t))$, where $f(0)=0$. The goal of the sector nonlinearity approach is to find the global sector such that $\nabla x(t)=f(x(t)) \in\left[\kappa_{1} \kappa_{2}\right] x(t)$ (see Fig. 1- (a)). This approach guarantees an exact T-S fuzzy model construction. However, note that it is sometimes difficult to find global sectors for general nonlinear systems. Thus, we consider local sector nonlinearity. This is reasonable as variables of physical systems are always bounded. Fig.1- (b) shows the local sector nonlinearity, where two lines become the local sectors under $x(t) \in\left[t_{1}, t_{2}\right]$. The T-S fuzzy model exactly represents the nonlinear system in the "local" region, that is, $x(t) \in\left[t_{1}, t_{2}\right]$, which is described as follows:

$$
\nabla x(t)=f(x(t))=\sum_{i=1}^{2} \theta_{i}(t) a_{i} x(t),
$$

where

$$
\begin{aligned}
& \theta_{1}(t)=\frac{w_{2}}{w_{1}+w_{2}}=\frac{f(x(t))-a_{2} x(t)}{a_{1} x(t)-a_{2} x(t)}, \\
& \theta_{2}(t)=\frac{w_{1}}{w_{1}+w_{2}}=\frac{a_{1} x(t)-f(x(t))}{a_{1} x(t)-a_{2} x(t)} .
\end{aligned}
$$

In addition, we can claim that $\theta_{1}(t)+\theta_{2}(t)=1$., $\theta_{1}(t) \geq 0$, and $\theta_{2}(t) \geq 0$. 


\section{Saturation Nonlinearity:}

Saturation nonlinearity is usually caused by limits on component size, properties of materials, and available power. Most actuators present saturation characteristics. For example, the output torque of a two-phase servomotor cannot increase infinitely and tends to saturate, due to the properties of the magnetic material. Similarly, valve-controlled hydraulic servomotors are saturated by the maximum flow rate. To address the saturation problem, three methods are exploited: the circle method (which basically deals with saturation as a sector-bounded nonlinearity), the so-called linear analysis method which consists in determining a region in which a linear controller does not saturate, and the polytopic representation method proposed by $\mathrm{Hu}$ and Lin (2001). This chapter will introduce the third method.

Consider the following saturation function:

$$
f(u(t))=\operatorname{sat}(u(t), \bar{u}),
$$

where the notation $\operatorname{sat}(u, \bar{u})$ means

$$
\operatorname{sat}(u, \bar{u})=\left[s_{1} \cdots s_{n_{u}}\right]^{T}, s_{i}=\operatorname{sign}\left(u_{i}\right) \min \left\{\bar{u}_{i},\left|u_{i}\right|\right\},
$$

in which $\bar{u} \in \mathcal{R}^{n_{u}}$ denotes the saturation level, sign returns the signs of the corresponding argument, and $u_{i}$ and $\bar{u}_{i}$ denote the $i$-th element of $u \in \mathcal{R}^{n_{u}}$ and $\bar{u} \in \mathcal{R}^{n_{u}}$, respectively. From the following lemma ( $\mathrm{Hu} \& \mathrm{Lin}, 2001)$, we can obtain a T-S fuzzy model for the saturation nonlinearity:

Lemma 1 Let $\mathcal{G}$ be the set of $n_{u} \times n_{u}$ diagonal matrices whose diagonal elements are 1 or 0 . Suppose that $\left|v_{i}\right| \leq \bar{u}_{i}$ for all $i=1, \cdots, n_{u}$, where $v_{i}$ and $\bar{u}_{i}$ denote the $i$-th element of $v \in \mathcal{R}^{n_{u}}$ and $\bar{u} \in \mathcal{R}^{n_{u}}$, respectively. Then

$$
\operatorname{sat}(u(t), \bar{u})=\sum_{i=1}^{2^{n_{u}}} \theta_{i}(t)\left(G_{i} u(t)+\bar{G}_{i} v(t)\right), \sum_{i=1}^{2^{n_{u}}} \theta_{i}(t)=1, \theta_{i}(t) \geq 0,
$$

where $G_{i}$ denote all elements of $\mathcal{G}, \bar{G}_{i}=I-G_{i}$.

In particular, for the case of $n_{u}=1$, the parameters are given as

$$
\theta_{1}(t)=\frac{\operatorname{sat}(u(t), \bar{u})-\left(G_{2} u(t)+\bar{G}_{2} v(t)\right)}{\left(G_{1}-G_{2}\right) u(t)+\left(\bar{G}_{1}-\bar{G}_{2}\right) v(t)}, \theta_{2}(t)=1-\theta_{1}(t) .
$$

\section{Fault-Related Nonlinearity:}

The actuator fault can be modeled as follows:

$$
f(u(t))=u^{F}(t)=\Lambda(t) u(t)
$$

where $\Lambda(t)=\operatorname{diag}\left\{\lambda_{1}(t), \lambda_{2}(t), \cdots, \lambda_{n_{u}}\right.$ with $\underline{\lambda}_{i} \leq \lambda_{i}(t) \leq \bar{\lambda}_{i} \leq 1, \quad i \in\left[1, n_{u}\right]$. Obviously, when $0 \leq \underline{\lambda}_{i}<\bar{\lambda}_{i} \leq 1$, it corresponds to the case of partial fault of the $i$-th actuator. When $\underline{\lambda}_{i}=\bar{\lambda}_{i}=1$, it implies that there is no fault in the $i$-th actuator. Define that the matrix set 


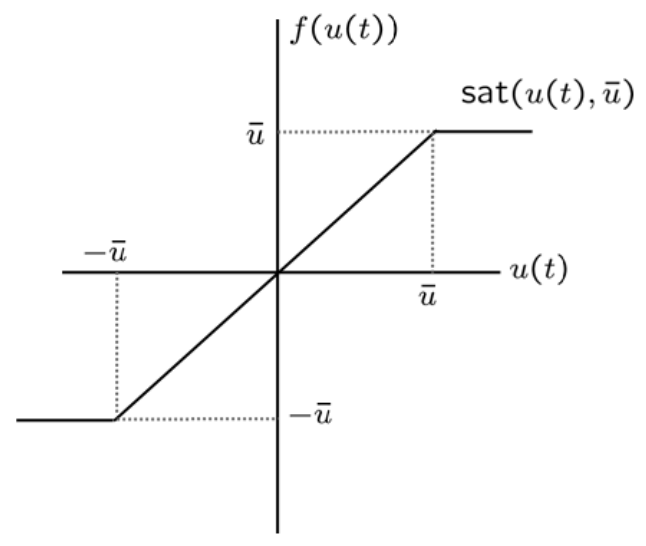

Fig. 2. Saturation nonlinearity.

$$
\mathcal{S}=\left\{\Lambda_{i} \mid \Lambda_{i}=\operatorname{diag}\left\{\lambda_{i 1}, \lambda_{i 2}, \cdots, \lambda_{i n_{u}}\right\}, \lambda_{i j}=\bar{\lambda}_{i} \text { or } \underline{\lambda}_{i}, j \in\left[1, n_{u}\right]\right\},
$$

where $i=1,2, \cdots, 2^{n_{u}-l}$ and $l\left(\leq n_{u}\right)$ is the number of the actuators without faults. Then, it is obvious that

$$
f(u(t))=\sum_{i=1}^{2^{n_{u}}-l} \theta_{i}(t) \Lambda_{i} u(t) .
$$

Here, let us assume that $u^{F}(t)$ is measurable. Then, in the case of $n_{u}-\ell=1$, the parameters can be calculated as follows:

$$
\theta_{1}(t)=\frac{u^{F}(t)-\Lambda_{2} u(t)}{\left(\Lambda_{1}-\Lambda_{2}\right) u(t)}, \theta_{2}(t)=\frac{\Lambda_{1}(t)-u^{F}(t)}{\left(\Lambda_{1}-\Lambda_{2}\right) u(t)}
$$

Remark 2: Since the actuator fault often act as the source of instability in many control systems, the study of reliable control has recently received a considerable amount of attention in control engineering. In particular, three different approaches to address the actuator fault problem have appeared in the literature from the 1980s: pole region assignment (Zhao \& Jiang, 1998), algebraic Riccati equation approach (Yang et al, 2001), and linear matrix inequality (LMI) approach (Liao et al, 2002; Wu \& Zhang, 2006), which achieve various reliability goals for linear systems.

\section{PLMI description and relaxation technique}

Solving the PLMIs is solving an infinite number of LMIs and is an extremely difficult problem. To overcome it, we shall take all the fuzzy weighting-dependent variables to be of polynomially parameter dependent structure, and then we shall propose an efficient relaxation technique (Kim \& Park, 2008; and Kim et al, 2009) that can replace the PLMIs into a finite number of LMIs. 


\section{A. Parameterized Linear Matrix Inequality (PLMI)}

The LMI technique is well-known as a unifying framework for formulating and solving problems in control theory. The main advantage of this technique is that complicated control problem can be solved very efficiently with interior point methods (Nesterov \& Nemirovski, 1994). A simple feasibility problem in semi-definite programming (SDP) is to find a solution to the following LMI:

$$
F(x)=F_{0}+\sum_{i=1}^{s} x_{i} F_{i}<0
$$

where the $x_{i}$ 's are the decision variables and the $F_{i}$ 's are given real symmetric matrices. A more complicated generalization of (25) has the following form:

$$
F(x(\theta))=F_{0}(\theta)+\sum_{i=1}^{s} x_{i}(\theta) F_{i}(\theta)<0,
$$

where $\theta \in \mathcal{R}^{r}$ is an additional parameter allowed to take any value in a compact set $\Gamma$ (the compact $\Gamma$ is typically polytopic). One calls (26) parameterized linear matrix inequality (PLMI) to stress the connection with the LMI control theory literature. The goal of (26) is to find $x_{i}(\theta)$ such that (26) holds for any admissible value of $\theta$, but it is very difficult to numerically solve the PLMI in (26) because of the following:

1. It is infinite-dimensional since the $x_{i}(\theta)$ are obtained in the infinite- dimensional space of the functions of $\theta$.

2. This is an infinitely constrained LMI problem for which each constraint corresponds to a given point in the range of $\theta$.

Thus, to overcome the difficulties arising from dimensionality, one needs a systematic technique that can turn an PLMI problem into a standard LMI problem. Motivated by the concern, we shall also deal with the problem by selecting an appropriate structure for the parameter-dependent variable $\mathcal{X}(\theta)$ to find a finite number of solvable LMIs from the PLMI (refer to Section 1).

\section{B. Relaxation technique}

In the case of adopting the structure in (1) for the analysis and synthesis of T-S fuzzy systems, the relaxation technique plays an important role in finding a less conservative set of solutions since the stability and stabilization conditions are generally of the following structure:

$$
\sum_{i_{1}=1}^{r} \cdots \sum_{i_{p}=1}^{r} \theta_{i_{1}}(t) \cdots \theta_{i_{p}}(t) \mathcal{L}_{i_{1} \cdots i_{p}}>0, \sum_{i=1}^{r} \theta_{i}(t)=1, \theta_{i}(t) \geq 0, i=1, \cdots, r .
$$

Without loss of generality, the following two statements are equivalent in the case where $p=1$ : 


$$
\begin{gathered}
\text { i) } \sum_{i=1}^{r} \theta_{i}(t) \mathcal{L}_{i}>0, \text { subject to } \sum_{i=1}^{r} \theta_{i}(t)=1, \theta_{i}(t) \geq 0, \\
\text { ii) } \mathcal{L}_{i}>0, \forall i=1, \cdots, r .
\end{gathered}
$$

However, in the case where $p=2$ :

$$
\sum_{i_{1}=1}^{r} \sum_{i_{2}=1}^{r} \theta_{i_{1}}(t) \theta_{i_{2}}(t) \mathcal{L}_{i_{1} i_{2}}>0, \text { subject to } \sum_{i_{1}=1}^{r} \theta_{i_{1}}(t)=1, \theta_{i_{1}}(t) \geq 0,
$$

we cannot conclude that (30) is equivalent to

$$
\mathcal{L}_{i_{1} i_{2}}>0, \forall i_{1}, i_{2}=1, \cdots, r .
$$

Of course, if (31) holds, then (30) also holds, but (31) leads to very conservative results with respect to (30). Thus, to reduce the conservatism caused by (31), various relaxation schemes have appeared in the literature (Tanaka et al, 1998; Kim \& Lee, 2000; Xiaodong \& Qingling, 2003; Tuan et al, 2001; Teixeira, 2003; Sala \& Ariño , 2007; Fang et al, 2006).

In this section, we shall introduces an useful relaxation technique for Cases 1 to 3 , which is made by incorporating some additional constraints on parameters into the interactions among the T-S fuzzy subsystems.

Henceforth, for a simple description, we use the following notations: $\theta_{i}=\theta_{i}(t), \dot{\theta}_{i}=\dot{\theta}_{i}(t)$, $\theta_{i}^{-}=\theta_{i}(t-1)$, and $\theta_{i}^{+}=\theta_{i}(t+1)$.

\section{Case 1 (Continuous-time):}

Consider the following codition with quadratic dependence for $\theta_{i}(t)$ :

$$
0<\mathcal{L}(\theta(t)) \stackrel{\Delta}{=} \mathcal{L}_{0}+\sum_{i=1}^{r} \theta_{i}\left(\mathcal{L}_{i}+\mathcal{L}_{i}^{T}\right)+\sum_{i=1}^{r} \theta_{i}^{2} \mathcal{L}_{i i}+\sum_{i=1}^{r}\left(\sum_{j=1}^{i-1} \theta_{i} \theta_{j} \mathcal{L}_{i j}+\sum_{j=i+1}^{r} \theta_{i} \theta_{j} \mathcal{L}_{i j}^{T}\right)
$$

subject to, for $i, j \in[1, r], j \neq i$,

$$
\text { (C1) } \sum_{i=1}^{r} \theta_{i}=1,(\mathrm{C} 2) 0 \leq \theta_{i} \leq \beta_{i},(\mathrm{C} 3) 0 \leq \theta_{i} \theta_{j} \text {. }
$$

By the S-procedure (Boyd et al, 1994) and Finsler's lemma (de Oliveira \& Skelton, 2001; Fang et al, 2004), the condition in Case 1 can be written as follows:

$$
0<\mathcal{L}(\theta(t))-\mathcal{N}(\theta(t))
$$

where $0 \leq \mathcal{N}(\theta(t))$ is given by

$$
\mathcal{N}(\theta(t))=\mathcal{C}_{1}+\mathcal{C}_{1}^{T}+\sum_{i=1}^{r} \mathcal{C}_{2 i}\left(\Lambda_{i}+\Lambda_{i}^{T}\right)+\sum_{i=1}^{r} \sum_{j=1, j \neq i}^{r} \mathcal{C}_{3 i j}\left(\Xi_{i j}+\Xi_{i j}^{T}\right),
$$

in which $\mathcal{C}_{1}, \mathcal{C}_{2 i}$, and $\mathcal{C}_{3 i j}$ are from (C1), (C2), and (C3), respectively: 


$$
\begin{aligned}
0=\mathcal{C}_{1} \triangleq\left[\begin{array}{c}
I \\
\theta_{1} I \\
\vdots \\
\theta_{r} I
\end{array}\right]^{T}\left[\begin{array}{c}
I \\
-I \\
\vdots \\
-I
\end{array}\right]\left[\begin{array}{llll}
S_{0} & S_{1} & \cdots & S_{r}
\end{array}\right]\left[\begin{array}{c}
I \\
\theta_{1} I \\
\vdots \\
\theta_{r} I
\end{array}\right], \\
0 \leq \mathcal{C}_{2 i} \triangleq-\theta_{i}^{2}+\beta_{i} \theta_{i}, 0 \leq \mathcal{C}_{3 i j} \triangleq \theta_{i} \theta_{j} .
\end{aligned}
$$

Here, note that the relaxation variables $S_{0}, S_{i}, \Lambda_{i}$, and $\Xi_{i j}$ are in $\mathcal{R}^{n_{c} \times n_{c}}$ and should satisfy that $0<\Lambda_{i}+\Lambda_{i}^{T}$ and $0<\Xi_{i j}+\Xi_{i j}^{T}$. Further, with some algebraic manipulations, the constraint, $0 \leq \mathcal{N}(\theta(t))$, can be rewritten as follows:

$$
0 \leq \mathcal{N}(\theta(t))=N_{0}+\sum_{i=1}^{r} \theta_{i}\left(N_{i}+N_{i}^{T}\right)+\sum_{i=1}^{r} \theta_{i}^{2} N_{i i}+\sum_{i=1}^{r}\left(\sum_{j=1}^{i-1} \theta_{i} \theta_{j} N_{i j}+\sum_{j=i+1}^{r} \theta_{i} \theta_{j} N_{i j}^{T}\right)
$$

where $\quad N_{0}=S_{0}+S_{0}^{T}, \quad N_{i}=\beta_{i} \Lambda_{i}-S_{0}+S_{i}, \quad N_{i i}=-\left(\Lambda_{i}+\Lambda_{i}^{T}\right)-\left(S_{i}+S_{i}^{T}\right), \quad N_{i j}=-\left(S_{i}+S_{j}\right)$ $+\left(\Xi_{i j}+\Xi_{j i}\right)$. Hence, the condition in (35) becomes, for all $\ell, m \in[1, r]$,

$$
0<\Gamma_{0}+\sum_{i=1}^{r} \theta_{i}\left(\Gamma_{i}+\Gamma_{i}^{T}\right)+\sum_{i=1}^{r} \theta_{i}^{2} \Delta_{i}+\sum_{i=1}^{r}\left(\sum_{j=1}^{i-1} \theta_{i} \theta_{j} \Phi_{i j}+\sum_{j=i+1}^{r} \theta_{i} \theta_{j} \Phi_{i j}^{T}\right)
$$

where

$$
\left\{\begin{array}{l}
\Gamma_{0}=\mathcal{L}_{0}-N_{0}=\mathcal{L}_{0}-S_{0}-S_{0}^{T} \\
\Gamma_{i}=\mathcal{L}_{i}-N_{i}=\mathcal{L}_{i}-\beta_{i} \Lambda_{i}+S_{0}-S_{i} \\
\Delta_{i}=\mathcal{L}_{i i}-N_{i i}=\mathcal{L}_{i i}+\left(\Lambda_{i}+\Lambda_{i}^{T}\right)+\left(S_{i}+S_{i}^{T}\right) \\
\Phi_{i j}=\mathcal{L}_{i j}-N_{i j}=\mathcal{L}_{i j}+\left(S_{i}+S_{j}\right)-\left(\Xi_{i j}+\Xi_{j i}\right)
\end{array}\right.
$$

As a result, the condition in (40) boils down to

$$
0<\left[\begin{array}{llll}
I & \theta_{1} I & \cdots & \theta_{r} I
\end{array}\right] \tilde{\mathcal{L}}\left[\begin{array}{llll}
I & \theta_{1} I & \cdots & \theta_{r} I
\end{array}\right]^{T}
$$

where

$$
\tilde{\mathcal{L}}=\left[\begin{array}{c:cccc}
\Gamma_{0} & (*) & (*) & \cdots & (*) \\
\hdashline \Gamma_{1} & \Delta_{1} & (*) & \cdots & (*) \\
\Gamma_{2} & \Phi_{21} & \Delta_{2} & \ddots & \vdots \\
\vdots & \vdots & \ddots & \ddots & (*) \\
\Gamma_{r} & \Phi_{r 1} & \cdots & \Phi_{r(r-1)} & \Delta_{r}
\end{array}\right] .
$$


Proposition 1 The condition in (33) subject to (C1) and (C2) holds if the following conditions hold: for all $i, j \in[1, r], j \neq i$,

$$
0<\tilde{\mathcal{L}}, 0<\Lambda_{i}+\Lambda_{i}^{T}, 0<\Xi_{i j}+\Xi_{i j}^{T}
$$

\section{Case 2 (Continuous-time):}

Consider the following condition with quadratic dependence $\theta_{i}(t)$ and $\dot{\theta}_{i}(t)$ :

$$
\begin{gathered}
0<\mathcal{L}(\theta(t), \dot{\theta}(t)) \triangleq \mathcal{L}_{0}+\sum_{i=1}^{r} \theta_{i}\left(\mathcal{L}_{i}+\mathcal{L}_{i}^{T}\right)+\sum_{i=1}^{r} \dot{\theta}_{i}\left(\breve{\mathcal{L}}_{i}+\breve{\mathcal{L}}_{i}^{T}\right)+\sum_{i=1}^{r} \theta_{i}^{2} \mathcal{L}_{i i} \\
+\sum_{i=1}^{r} \dot{\theta}_{i}^{2} \breve{\mathcal{L}}_{i i}+\sum_{i=1}^{r} \sum_{j=1}^{r} \dot{\theta}_{i} \theta_{j}\left(\overline{\mathcal{L}}_{i j}+\overline{\mathcal{L}}_{i j}^{T}\right)+\sum_{i=1}^{r}\left(\sum_{j=1}^{i-1} \theta_{i} \theta_{j} \mathcal{L}_{i j}+\sum_{j=i+1}^{r} \theta_{i} \theta_{j} \mathcal{L}_{i j}^{T}\right) \\
+\sum_{i=1}^{r}\left(\sum_{j=1}^{i-1} \dot{\theta}_{i} \dot{\theta}_{j} \breve{\mathcal{L}}_{i j}+\sum_{j=i+1}^{r} \dot{\theta}_{i} \dot{\theta}_{j} \breve{\mathcal{L}}_{i j}^{T}\right)
\end{gathered}
$$

subject to, for $i, j \in[1, r], j \neq i$,

$$
\text { (C1) } \sum_{i=1}^{r} \theta_{i}=1,(C 2) 0 \leq \theta_{i} \leq \beta_{i},(C 3) 0 \leq \theta_{i} \theta_{j},(C 4) \rho_{i} \leq \dot{\theta}_{i} \leq v_{i} \text {. }
$$

By the S-procedure (Boyd et al, 1994) and Finsler's lemma (de Oliveira \& Skelton, 2001; Fang et al, 2004), the condition in Case 2 can be written as follows:

$$
0<\mathcal{L}(\theta(t), \dot{\theta}(t))-\mathcal{N}(\theta(t), \dot{\theta}(t)),
$$

where $0 \leq \mathcal{N}(\theta(t), \dot{\theta}(t))$ is given by

$$
\mathcal{N}(\theta(t), \dot{\theta}(t))=\mathcal{C}_{1}+\mathcal{C}_{1}^{T}+\sum_{i=1}^{r} \mathcal{C}_{2 i}\left(\Lambda_{i}+\Lambda_{i}^{T}\right)+\sum_{i=1}^{r} \sum_{j=1, j \neq i}^{r} \mathcal{C}_{3 i j}\left(\Xi_{i j}+\Xi_{i j}^{T}\right)+\sum_{i=1}^{r} \mathcal{C}_{4 i}\left(Z_{i}+Z_{i}^{T}\right),
$$

in which $\mathcal{C}_{1}, \mathcal{C}_{2 i}, \mathcal{C}_{3 i j}$, and $\mathcal{C}_{4 i}$ are from (C1), (C2), (C3), and (C4), respectively:

$$
\begin{gathered}
0=\mathcal{C}_{1} \triangleq\left[\begin{array}{c}
I \\
\theta_{1} I \\
\vdots \\
\theta_{r} I
\end{array}\right]^{T}\left[\begin{array}{c}
I \\
-I \\
\vdots \\
-I
\end{array}\right]\left[\begin{array}{llll}
S_{0} & S_{1} & \cdots & S_{r}
\end{array}\right]\left[\begin{array}{c}
I \\
\theta_{1} I \\
\vdots \\
\theta_{r} I
\end{array}\right], \\
0 \leq \mathcal{C}_{2 i} \triangleq-\theta_{i}^{2}+\beta_{i} \theta_{i}, 0 \leq \mathcal{C}_{3 i j} \triangleq \theta_{i} \theta_{j}, 0 \leq \mathcal{C}_{4 i} \triangleq-\dot{\theta}_{i}^{2}+\left(\rho_{i}+v_{i}\right) \dot{\theta}_{i}-\rho_{i} v_{i} .
\end{gathered}
$$

Here, note that the multiplier variables $S_{0}, S_{i}, \Lambda_{i}, Z_{i}$ and $\Xi_{i j}$ are in $\mathcal{R}^{n_{c} \times n_{c}}$ and should satisfy that $0<\Lambda_{i}+\Lambda_{i}^{T}, 0<Z_{i}+Z_{i}^{T}$ and $0<\Xi_{i j}+\Xi_{i j}^{T}$. Further, with some algebraic manipulations, the constraint $0 \leq \mathcal{N}(\theta(t))$ can be represented as follows: 


$$
\begin{gathered}
0 \leq \mathcal{N}(\theta(t), \dot{\theta}(t))=N_{0}+\sum_{i=1}^{r} \theta_{i}\left(N_{i}+N_{i}^{T}\right)+\sum_{i=1}^{r} \dot{\theta}_{i}\left(N_{i}+N_{i}^{T}\right)+\sum_{i=1}^{r} \theta_{i}^{2} N_{i i} \\
+\sum_{i=1}^{r} \dot{\theta}_{i}^{2} N_{i i}+\sum_{i=1}^{r}\left(\sum_{j=1}^{i-1} \theta_{i} \theta_{j} N_{i j}+\sum_{j=i+1}^{r} \theta_{i} \theta_{j} N_{i j}^{T}\right),
\end{gathered}
$$

where $\quad N_{0}=S_{0}+S_{0}^{T}-\sum_{i=1}^{r} \rho_{i} v_{i}\left(Z_{i}+Z_{i}^{T}\right), \quad N_{i}=\beta_{i} \Lambda_{i}-S_{0}+S_{i}, \quad N_{i}=\left(\rho_{i}+v_{i}\right) Z_{i}$, $N_{i i}=-\left(\Lambda_{i}+\Lambda_{i}^{T}\right)-\left(S_{i}+S_{i}^{T}\right), \quad N_{i i}=-\left(Z_{i}+Z_{i}^{T}\right)$, and $N_{i j}=-\left(S_{i}+S_{j}\right)+\left(\Xi_{i j}+\Xi_{j i}\right)$. Hence, the condition in (47) becomes, for all $\ell, m \in[1, r]$,

$$
\begin{gathered}
0<\Gamma_{0}+\sum_{i=1}^{r} \theta_{i}\left(\Gamma_{i}+\Gamma_{i}^{T}\right)+\sum_{i=1}^{r} \dot{\theta}_{i}\left(\breve{\Gamma}_{i}+\breve{\Gamma}_{i}^{T}\right)+\sum_{i=1}^{r} \theta_{i}^{2} \Delta_{i} \\
+\sum_{i=1}^{r} \dot{\theta}_{i}^{2} \breve{\Delta}_{i}+\sum_{i=1}^{r} \sum_{j=1}^{r} \dot{\theta}_{i} \theta_{j}\left(\Pi_{i j}+\Pi_{i j}^{T}\right)+\sum_{i=1}^{r}\left(\sum_{j=1}^{i-1} \theta_{i} \theta_{j} \Phi_{i j}+\sum_{j=i+1}^{r} \theta_{i} \theta_{j} \Phi_{i j}^{T}\right) \\
+\sum_{i=1}^{r}\left(\sum_{j=1}^{i-1} \dot{\theta}_{i} \dot{\theta}_{j} \breve{\Phi}_{i j}+\sum_{j=i+1}^{r} \dot{\theta}_{i} \dot{\theta}_{j} \breve{\Phi}_{i j}^{T}\right),
\end{gathered}
$$

where

$$
\left\{\begin{array}{l}
\Gamma_{0}=\mathcal{L}_{0}-N_{0}=\mathcal{L}_{0}-S_{0}-S_{0}^{T}+\sum_{i=1}^{r} \rho_{i} v_{i}\left(Z_{i}+Z_{i}^{T}\right) \\
\Gamma_{i}=\mathcal{L}_{i}-N_{i}=\mathcal{L}_{i}-\beta_{i} \Lambda_{i}+S_{0}-S_{i} \\
\breve{\Gamma}_{i}=\breve{\mathcal{L}}_{i}-N_{i}=\breve{\mathcal{L}}_{i}-\left(\rho_{i}+v_{i}\right) Z_{i} \\
\Delta_{i}=\mathcal{L}_{i i}-N_{i i}=\mathcal{L}_{i i}+\left(\Lambda_{i}+\Lambda_{i}^{T}\right)+\left(S_{i}+S_{i}^{T}\right) \\
\breve{\Delta}_{i}=\breve{\mathcal{L}}_{i i}-N_{i i}=\breve{\mathcal{L}}_{i i}+Z_{i}+Z_{i}^{T} \\
\Phi_{i j}=\mathcal{L}_{i j}-N_{i j}=\mathcal{L}_{i j}+\left(S_{i}+S_{j}\right)-\left(\Xi_{i j}+\Xi_{j i}\right) \\
\breve{\Phi}_{i j}=\breve{\mathcal{L}}_{i j} \\
\Pi_{i j}=\overline{\mathcal{L}}_{i j}
\end{array} .\right.
$$

As a result, the condition (51) boils down to

$$
0<\left[\begin{array}{lllllll}
I & \theta_{1} I & \cdots & \theta_{r} I & \dot{\theta}_{1} I & \cdots & \dot{\theta}_{r} I
\end{array}\right] \tilde{\mathcal{L}}\left[\begin{array}{llllllll}
I & \theta_{1} I & \cdots & \theta_{r} I & \dot{\theta}_{1} I & \cdots & \dot{\theta}_{r} I
\end{array}\right]^{T},
$$

where 


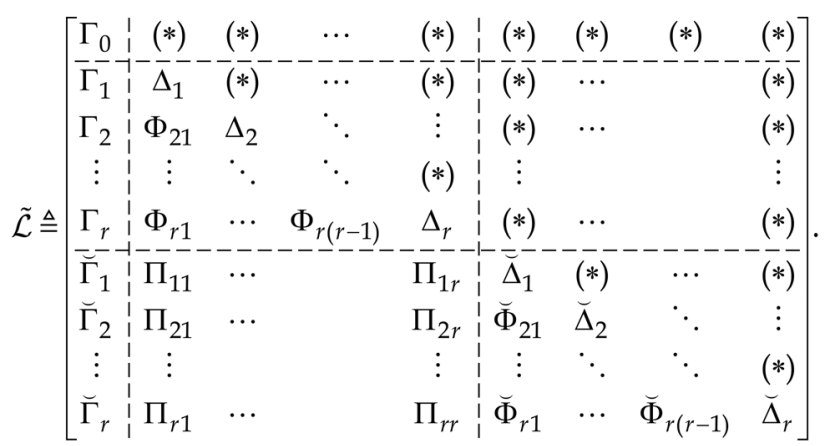

Proposition 2 The condition in (45) subject to (C1) - (C4) holds if the following conditions hold: for all $i, j \in[1, r], j \neq i$,

$$
0<\tilde{\mathcal{L}}, 0<\Lambda_{i}+\Lambda_{i}^{T}, 0<\Xi_{i j}+\Xi_{i j}^{T}, 0<Z_{i}+Z_{i}^{T} .
$$

\section{Case 3 (Discrete-time):}

Consider the following condition with quadratic dependence $\theta_{i}(t)$ and $\dot{\theta}_{i}(t-1)$ :

$$
\begin{array}{r}
0<\mathcal{L}(\theta(t-1), \theta(t)) \triangleq \mathcal{L}_{0}+\sum_{i=1}^{r} \theta_{i}\left(\mathcal{L}_{i}+\mathcal{L}_{i}^{T}\right)+\sum_{i=1}^{r} \theta_{i}^{-}\left(\breve{\mathcal{L}}_{i}+\breve{\mathcal{L}}_{i}^{T}\right)+\sum_{i=1}^{r} \theta_{i}^{2} \mathcal{L}_{i i} \\
+\sum_{i=1}^{r}\left(\theta_{i}^{-}\right)^{2} \breve{\mathcal{L}}_{i i}+\sum_{i=1}^{r} \sum_{j=1}^{r} \theta_{i}^{-} \theta_{j}\left(\overline{\mathcal{L}}_{i j}+\overline{\mathcal{L}}_{i j}^{T}\right)+\sum_{i=1}^{r}\left(\sum_{j=1}^{i-1} \theta_{i} \theta_{j} \mathcal{L}_{i j}+\sum_{j=i+1}^{r} \theta_{i} \theta_{j} \mathcal{L}_{i j}^{T}\right) \\
+\sum_{i=1}^{r}\left(\sum_{j=1}^{i-1} \theta_{i}^{-} \theta_{j}^{-} \breve{\mathcal{L}}_{i j}+\sum_{j=i+1}^{r} \theta_{i}^{-} \theta_{j}^{-} \breve{\mathcal{L}}_{i j}^{T}\right)
\end{array}
$$

subject to, for $i, j \in[1, r], j \neq i$,

$$
\begin{gathered}
\text { (C1) } \sum_{i=1}^{r} \theta_{i}=1, \sum_{i=1}^{r} \theta_{i}^{-}=1,(\mathrm{C} 2) 0 \leq \theta_{i} \leq \beta_{i}, 0 \leq \theta_{i}^{-} \leq \beta_{i}, \\
\text { (C3) } 0 \leq \theta_{i} \theta_{j}, 0 \leq \theta_{i}^{-} \theta_{j}^{-},(\mathrm{C} 4)\left|\theta_{i}-\theta_{i}^{-}\right| \leq \delta_{i} \leq 1 .
\end{gathered}
$$

By the S-procedure (Boyd et al, 1994) and Finsler's lemma (de Oliveira and Skelton, 2001; Fang et al, 2004), the condition in Case 3 can be written as follows:

$$
0<\mathcal{L}(\theta(t-1), \theta(t))-\mathcal{N}(\theta(t-1), \theta(t)),
$$

where $0 \leq \mathcal{N}(\theta(t-1), \theta(t))$ is given by

$$
\mathcal{N}(\theta(t-1), \theta(t))=\mathcal{C}_{1}+\mathcal{C}_{1}^{T}+\sum_{i=1}^{r} \mathcal{C}_{2 i}\left(\Lambda_{i}+\Lambda_{i}^{T}\right)+\sum_{i=1}^{r} \breve{\mathcal{C}}_{2 i}\left(\breve{\Lambda}_{i}+\breve{\Lambda}_{i}^{T}\right)
$$




$$
+\sum_{i=1}^{r} \sum_{j=1, j \neq i}^{r} \mathcal{C}_{3 i j}\left(\Xi_{i j}+\Xi_{i j}^{T}\right)+\sum_{i=1}^{r} \sum_{j=1, j \neq i}^{r} \breve{\mathcal{C}}_{3 i j}\left(\breve{\Xi}_{i j}+\breve{\Xi}_{i j}^{T}\right)+\sum_{i=1}^{r} \mathcal{C}_{4 i}\left(Z_{i}+Z_{i}^{T}\right),
$$

in which $\mathcal{C}_{1}, \mathcal{C}_{2 i}, \breve{\mathcal{C}}_{2 i}, \mathcal{C}_{3 i j}, \breve{\mathcal{C}}_{3 i j}$ and $\mathcal{C}_{4 i}$ are from (C1)-(C4):

$$
\begin{gathered}
0=\mathcal{C}_{1} \triangleq\left[\begin{array}{c}
I \\
\theta_{1} I \\
\vdots \\
\theta_{r} I \\
\theta_{1}^{-} I \\
\vdots \\
\theta_{r}^{-} I
\end{array}\right]^{T}\left[\begin{array}{cc}
I & I \\
-I & 0 \\
\vdots & \vdots \\
-I & 0 \\
0 & -I \\
\vdots & \vdots \\
0 & -I
\end{array}\right]\left[\begin{array}{cc}
X_{0}^{T} & Y_{0}^{T} \\
X_{1}^{T} & Y_{1}^{T} \\
\vdots & \vdots \\
X_{r}^{T} & Y_{r}^{T} \\
R_{1} & S_{1} \\
\vdots & \vdots \\
R_{r} & S_{r}
\end{array}\right]^{T}\left[\begin{array}{c}
I \\
\theta_{1} I \\
\vdots \\
\theta_{r} I \\
\theta_{1}^{-} I \\
\vdots \\
\theta_{r}^{-} I
\end{array}\right], \\
0 \leq \mathcal{C}_{2 i} \triangleq-\theta_{i}^{2}+\beta_{i} \theta_{i}, 0 \leq \breve{\mathcal{C}}_{2 i} \triangleq-\left(\theta_{i}^{-}\right)^{2}+\beta_{i} \theta_{i}^{-}, \\
0 \leq \mathcal{C}_{3 i j} \triangleq \theta_{i} \theta_{j}, 0 \leq \breve{\mathcal{C}}_{3 i j} \triangleq \theta_{i}^{-} \theta_{j}^{-}, 0 \leq \mathcal{C}_{4 i} \triangleq \delta_{i}^{2}-\theta_{i}^{2}+2 \theta_{i} \theta_{i}^{-}-\left(\theta_{i}^{-}\right)^{2} .
\end{gathered}
$$

Here, note that the multiplier variables $X_{0}, X_{i}, Y_{0}, Y_{i}, R_{i}, S_{i}, \Lambda_{i}, \breve{\Lambda}_{i}, \Xi_{i j}, \breve{\Xi}_{i j}$, and $Z_{i}$ are in $\mathcal{R}^{n_{c} \times n_{c}}$ and should satisfy that $0<\Lambda_{i}+\Lambda_{i}^{T}, 0<\breve{\Lambda}_{i}+\breve{\Lambda}_{i}^{T}, 0<\Xi_{i j}+\Xi_{i j}^{T}, 0<\breve{\Xi}_{i j}+\breve{\Xi}_{i j}^{T}$, and $0<Z_{i}+Z_{i}^{T}$. Further, with some algebraic manipulations, the constraint $0 \leq \mathcal{N}(\theta(t-1), \theta(t))$ can be rewritten as follows:

$$
\begin{gathered}
0 \leq \mathcal{N}(\theta(t-1), \theta(t))=N_{0}+\sum_{i=1}^{r} \theta_{i}\left(N_{i}+N_{i}^{T}\right)+\sum_{i=1}^{r} \theta_{i}^{-}\left(N_{i}+N_{i}^{T}\right)+\sum_{i=1}^{r} \theta_{i}^{2} N_{i i} \\
+\sum_{i=1}^{r}\left(\theta_{i}^{-}\right)^{2} N_{i i}+\sum_{i=1}^{r} \sum_{j=1}^{r} \theta_{i}^{-} \theta_{j}\left(N_{i j}+N_{i j}^{T}\right)+\sum_{i=1}^{r}\left(\sum_{j=1}^{i-1} \theta_{i} \theta_{j} N_{i j}+\sum_{j=i+1}^{r} \theta_{i} \theta_{j} N_{i j}^{T}\right) \\
+\sum_{i=1}^{r}\left(\sum_{j=1}^{i-1} \theta_{i}^{-} \theta_{j}^{-} N_{i j}+\sum_{j=i+1}^{r} \theta_{i}^{-} \theta_{j}^{-} N_{i j}^{T}\right),
\end{gathered}
$$

where

$$
\begin{gathered}
N_{0}=\left(X_{0}+X_{0}^{T}\right)+\left(Y_{0}+Y_{0}^{T}\right)+\sum_{i=1}^{r} \delta_{i}^{2}\left(Z_{i}+Z_{i}^{T}\right), N_{i}=X_{i}+Y_{i}-X_{0}+\beta_{i} \Lambda_{i}, \\
N_{i}=R_{i}+S_{i}-Y_{0}+\beta_{i} \breve{\Lambda}_{i}, N_{i i}=-\left(X_{i}+X_{i}^{T}\right)-\left(\Lambda_{i}+\Lambda_{i}^{T}\right)-\left(Z_{i}+Z_{i}^{T}\right), \\
N_{i i}=-\left(S_{i}+S_{i}^{T}\right)-\left(\breve{\Lambda}_{i}+\breve{\Lambda}_{i}^{T}\right)-\left(Z_{i}+Z_{i}^{T}\right), N_{i j}=\left\{\begin{array}{cc}
-R_{i}-Y_{i}+2 Z_{i}, & j=i \\
-R_{i}-Y_{j}, & j \neq i
\end{array}\right.
\end{gathered}
$$




$$
N_{i j}=-X_{i}-X_{j}+\Xi_{i j}+\Xi_{j i}, N_{i j}=-S_{i}-S_{j}+\breve{\Xi}_{i j}+\breve{\Xi}_{j i} .
$$

Hence, the condition (35) becomes, for all $\ell, m \in[1, r]$,

$$
\begin{gathered}
0<\Gamma_{0}+\sum_{i=1}^{r} \theta_{i}\left(\Gamma_{i}+\Gamma_{i}^{T}\right)+\sum_{i=1}^{r} \theta_{i}^{-}\left(\breve{\Gamma}_{i}+\breve{\Gamma}_{i}^{T}\right)+\sum_{i=1}^{r} \theta_{i}^{2} \Delta_{i} \\
+\sum_{i=1}^{r}\left(\theta_{i}^{2}\right)^{-} \breve{\Delta}_{i}+\sum_{i=1}^{r} \sum_{j=1}^{r} \theta_{i}^{-} \theta_{j}\left(\Pi_{i j}+\Pi_{i j}^{T}\right)+\sum_{i=1}^{r}\left(\sum_{j=1}^{i-1} \theta_{i} \theta_{j} \Phi_{i j}+\sum_{j=i+1}^{r} \theta_{i} \theta_{j} \Phi_{i j}^{T}\right) \\
+\sum_{i=1}^{r}\left(\sum_{j=1}^{i-1} \theta_{i}^{-} \theta_{j}^{-} \breve{\Phi}_{i j}+\sum_{j=i+1}^{r} \theta_{i}^{-} \theta_{j}^{-} \breve{\Phi}_{i j}^{T}\right),
\end{gathered}
$$

where $\Gamma_{0}=\mathcal{L}_{0}-N_{0}, \Gamma_{i}=\mathcal{L}_{i}-N_{i}, \breve{\Gamma}_{i}=\breve{\mathcal{L}}_{i}-N_{i}, \Delta_{i}=\mathcal{L}_{i i}-N_{i i}, \breve{\Delta}_{i}=\breve{\mathcal{L}}_{i i}-N_{i i}, \Phi_{i j}=\mathcal{L}_{i j}-N_{i j}$, $\breve{\Phi}_{i j}=\breve{\mathcal{L}}_{i j}-N_{i j}, \Pi_{i j}=\overline{\mathcal{L}}_{i j}-N_{i j}$. As a result, the condition in (62) boils down to

$$
0<\left[\begin{array}{lllllll}
I & \theta_{1} I & \cdots & \theta_{r} I & \theta_{1}^{-} I & \cdots & \theta_{r}^{-} I
\end{array}\right] \tilde{\mathcal{L}}\left[\begin{array}{lllllll}
I & \theta_{1} I & \cdots & \theta_{r} I & \theta_{1}^{-} I & \cdots & \theta_{r}^{-} I
\end{array}\right]^{T},
$$

where

$$
\tilde{\mathcal{L}} \triangleq\left[\begin{array}{c:cccc:cccc}
\Gamma_{0} & (*) & (*) & \cdots & (*) & (*) & (*) & (*) & (*) \\
\hdashline \Gamma_{1} & \Delta_{1} & (*) & \cdots & (*) & (*) & \cdots & & (*) \\
\Gamma_{2} & \Phi_{21} & \Delta_{2} & \ddots & \vdots & (*) & \cdots & (*) \\
\vdots & \vdots & \ddots & \ddots & (*) & \vdots & & \vdots \\
\Gamma_{r} & \Phi_{r 1} & \cdots & \Phi_{r(r-1)} & \Delta_{r} & (*) & \cdots & & (*) \\
\hdashline \Gamma_{1} & \Pi_{11} & \cdots & & \Pi_{1 r} & \breve{\Delta}_{1} & (*) & \cdots & (*) \\
\breve{\Gamma}_{2} & \Pi_{21} & \cdots & & \Pi_{2 r} & \breve{\Phi}_{21} & \breve{\Delta}_{2} & \ddots & \vdots \\
\vdots & \vdots & & & \vdots & \vdots & \ddots & \ddots & (*) \\
\breve{\Gamma}_{r} & \Pi_{r 1} & \cdots & & \Pi_{r r} & \breve{\Phi}_{r 1} & \cdots & \breve{\Phi}_{r(r-1)} & \breve{\Delta}_{r}
\end{array}\right] .
$$

Proposition 3 The condition in (55) subject to (C1) - (C4) holds if the following conditions hold: for all $i, j \in[1, r], j \neq i$,

$$
0<\tilde{\mathcal{L}}, 0<\Lambda_{i}+\Lambda_{i}^{T}, 0<\breve{\Lambda}_{i}+\breve{\Lambda}_{i}^{T}, 0<\Xi_{i j}+\Xi_{i j}^{T}, 0<\breve{\Xi}_{i j}+\breve{\Xi}_{i j}^{T}, 0<Z_{i}+Z_{i}^{T}
$$

\section{Stabilization of T-S fuzzy systems}

\section{A. Lyapunov function}

Based on the modeled T-S fuzzy systems (see Section 2), various feedback controllers have been recently developed under the well-known relaxation techniques (see Section 3), by taking advantage of Lyapunov functions, such as CQLF, PLF, and FWLF (refer to Section 1). The common quadratic Lyapunov function (CQLF) is given as follows: 


$$
V(x(t))=x^{T}(t) P x(t), P>0,
$$

and the fuzzy weighting-dependent Lyapunov function (FWLF) can be written as follows:

$$
V(x(t))=x^{T}(t) P(\theta(t)) x(t), P(\theta(t))>0 .
$$

As mentioned in Section 1, since, for a large number of T-S fuzzy subsystems $\left(A_{i}, B_{i}, C_{i}\right)$, the former approach leads to over-conservative design solutions, recent research efforts have focused on using the latter approach when establishing a feedback controller that ensures the stability of the closed-loop system.

When using the FWLF approach for stabilization of continuous-time T-S fuzzy systems, one needs to assume that the upper and lower bounds of $\dot{\theta}(t)$ are measurable and the matrix inverse is computed in real time. Since it is very strict to make the assumptions, one has made less use of the FWLF in continuous-time F-S fuzzy systems, compared with the discrete-time case. Furthermore, since it is impractical to fully measure the rate vector $\dot{\theta}(t)$, the controller should be constructed only by the current-time information on parameters, which leads to an inflexible result for more changeable T-S fuzzy systems. In the discretetime case, on the other hand, it is possible to make a feedback control law dependent not only on the current-time parameters but also the one-step-past parameters.

Now, let us consider the matrix $P(\theta(t))$ of the following form:

\section{FWLF with APD (FWLF-APD):}

$$
P(\theta(t))=\sum_{i=1}^{r} \theta_{i}(t) P_{i}, P_{i}>0, \sum_{i=1}^{r} \theta_{i}(t)=1, \theta_{i}(t) \geq 0, i=1, \cdots, r,
$$

FWLF with QPD (PDLF-QPD):

$$
P(\theta(t))=\sum_{i=1}^{r} \sum_{j=1}^{r} \theta_{i}(t) \theta_{j}(t) P_{i j}, P_{i j}>0, \sum_{i=1}^{r} \theta_{i}(t)=1, \theta_{i}(t) \geq 0, i=1, \cdots, r .
$$

\section{B. Stabilization of continuous-time T-S fuzzy systems}

Consider a T-S fuzzy system described by the following differential equation:

$$
\dot{x}(t)=A(\theta(t)) x(t)+B(\theta(t)) u(t),
$$

where $x(t) \in R^{n_{x}}$ and $u(t) \in R^{n_{u}}$ denote the state and control input, respectively; and

$$
A(\theta(t))=A_{0}+\sum_{i=1}^{r} \theta_{i}(t) A_{i}, B(\theta(t))=B_{0}+\sum_{i=1}^{r} \theta_{i}(t) B_{i} .
$$

For the stabilization of (69), consider the following fuzzy weighting-dependent statefeedback controller:

$$
u(t)=F(\theta(t)) x(t)
$$


Then, the closed-loop system under (71) is described as follows:

$$
\dot{x}(t)=(A(\theta(t))+B(\theta(t)) F(\theta(t))) x(t) .
$$

\section{Stabilization using CQLF:}

In the case where $\theta(t)$ is measurable with known bounds but the full information on $\dot{\theta}(t)$ is unknown, the CQLF approach is valuable and applicable to T-S fuzzy systems.

Theorem 1: (PLMI-based condition) Suppose that there exist matrices $\bar{P}>0$ and $\bar{F}(\theta(t))$ such that

$$
\bar{P} A^{T}(\theta(t))+\bar{F}^{T}(\theta(t)) B^{T}(\theta(t))+A(\theta(t)) \bar{P}+B(\theta(t)) \bar{F}(\theta(t))<0,
$$

where $\bar{P}=P^{-1}$ and $\bar{F}(\theta(t))=F(\theta(t)) \bar{P}$. Then, the closed-loop system in (72) is asymptotically stable for all admissible grades. Moreover, the controller is reconstructed as follows:

$$
F(\theta(t))=\bar{F}(\theta(t)) \bar{P}^{-1} .
$$

Proof The proof is straightforward, and hence is omitted here.

In the following, we shall derive a finite number of LMIs from (73). To this end, let us first consider

$$
\bar{F}(\theta(t))=\bar{F}_{0}+\sum_{i=1}^{r} \theta_{i}(t) \bar{F}_{i}
$$

Then, we can obtain the following theorem with the help of the relaxation technique in Case 1. Theorem 2: (LMI-based condition) Suppose that there exist matrices $\bar{P}>0, \bar{F}_{0}, \bar{F}_{i}, S_{0}, S_{i}, \Lambda_{i}$, and $\Xi_{i j}$ such that

$$
\begin{gathered}
0<\left[\begin{array}{c:cccc}
\Gamma_{0} & (*) & (*) & \cdots & (*) \\
\hdashline \Gamma_{1} & \Delta_{1} & (*) & \cdots & (*) \\
\Gamma_{2} & \Phi_{21} & \Delta_{2} & \ddots & \vdots \\
\vdots & \vdots & \ddots & \ddots & (*) \\
\Gamma_{r} & \Phi_{r 1} & \cdots & \Phi_{r(r-1)} & \Delta_{r}
\end{array}\right], \\
0 \leq \Lambda_{i}+\Lambda_{i}^{T}, 0 \leq \Xi_{i j}+\Xi_{j i}^{T}, \forall i, j \in[1, r], j \neq i .
\end{gathered}
$$

Then, the closed-loop system in (72) is asymptotically stable for all admissible grades satisfying the constraints (34). Moreover, the controller is given by

$$
F(\theta(t))=\left(\bar{F}_{0}+\sum_{i=1}^{r} \theta_{i}(t) \bar{F}_{i}\right) \bar{P}^{-1} .
$$

Proof The condition in (73) is equivalent to

$$
0<\mathcal{L}(\theta(t)) \triangleq-\left(\bar{P} A^{T}(\theta(t))+\bar{F}^{T}(\theta(t)) B^{T}(\theta(t))+A(\theta(t)) \bar{P}+B(\theta(t)) \bar{F}(\theta(t))\right)
$$


which can be rewritten by (70) and (75) into

$$
0<\mathcal{L}_{0}+\sum_{i=1}^{r} \theta_{i}(t)\left(\mathcal{L}_{i}+\mathcal{L}_{i}^{T}\right)+\sum_{i=1}^{r} \theta_{i}^{2}(t) \mathcal{L}_{i i}+\sum_{i=1}^{r}\left(\sum_{j=1}^{i-1} \theta_{i}(t) \theta_{j}(t) \mathcal{L}_{i j}+\sum_{j=i+1}^{r} \theta_{i}(t) \theta_{j}(t) \mathcal{L}_{i j}^{T}\right),
$$

where

$$
\begin{gathered}
\mathcal{L}_{0}=-\left(A_{0} \bar{P}+B_{0} \bar{F}_{0}+\bar{P} A_{0}^{T}+\bar{F}_{0}^{T} B_{0}^{T}\right), \mathcal{L}_{i}=-\left(A_{i} \bar{P}+B_{0} \bar{F}_{i}+B_{i} \bar{F}_{0}\right), \\
\mathcal{L}_{i i}=-\left(B_{i} \bar{F}_{i}+\bar{F}_{i}^{T} B_{i}^{T}\right), \mathcal{L}_{i j}=-\left(B_{i} \bar{F}_{j}+B_{j} \bar{F}_{i}\right) .
\end{gathered}
$$

Hence, reminding the relaxation technique in Case 1, we can clearly see that the condition $\mathcal{L}(\theta(t))>0$ subject to (34) is guaranteed by (76) and (77), where

$$
\begin{gathered}
\Gamma_{0}=\mathcal{L}_{0}-S_{0}-S_{0}^{T}, \Gamma_{i}=\mathcal{L}_{i}-\beta_{i} \Lambda_{i}+S_{0}-S_{i}, \\
\Delta_{i}=\mathcal{L}_{i i}+\left(\Lambda_{i}+\Lambda_{i}^{T}\right)+\left(S_{i}+S_{i}^{T}\right), \\
\Phi_{i j}=\mathcal{L}_{i j}+\left(S_{i}+S_{j}\right)-\left(\Xi_{i j}+\Xi_{j i}\right) .
\end{gathered}
$$

\section{Stabilization using FWLF-APD:}

Let us assume that $\theta(t)$ is measurable and the bounds of $\dot{\theta}(t)$ are given as

$$
\rho_{i} \leq \dot{\theta}_{i}(t) \leq v_{i}, \text { for } i=1, \cdots, r \text {. }
$$

Then, in this sense, we can consider a fuzzy weighting-dependent Lyapunov function $V(x(t))$ such as

$$
V(x(t))=x^{T}(t) P(\theta(t)) x(t), P(\theta(t))>0,
$$

based on which we can obtain the following PLMI-based stabilization condition.

Theorem 3: (PLMI-based condition) Suppose that there exist matrices $\bar{P}(\theta(t))>0, \dot{\bar{P}}(\theta(t))$, and $\bar{F}(\theta(t))$ such that

$$
\dot{\bar{P}}(\theta(t))>\bar{P}(\theta(t)) A^{T}(\theta(t))+\bar{F}^{T}(\theta(t)) B^{T}(\theta(t))+A(\theta(t)) \bar{P}(\theta(t))+B(\theta(t)) \bar{F}(\theta(t)),
$$

where $\bar{P}(\theta(t))=P^{-1}(\theta(t))$ and $\bar{F}(\theta(t))=F(\theta(t)) \bar{P}(\theta(t))$. Then, the closed-loop system in (72) is asymptotically stable for all admissible grades. Moreover, the controller is given by

$$
F(\theta(t))=\bar{F}(\theta(t)) \bar{P}^{-1}(\theta(t)) .
$$

Proof The proof is straightforward, and hence is omitted here.

In the following, we shall derive a finite number of LMIs from (87). To this end, let us take 


$$
\bar{P}(\theta(t))=\bar{P}+\sum_{i=1}^{r} \theta_{i}(t) \bar{P}_{i}, \bar{P}>0, \bar{P}_{i}>0 .
$$

Then, we can obtain the following theorem with the help of the relaxation scheme in Case 2. Theorem 4: (LMI-based condition) Suppose that there exist matrices $\bar{P}>0, \bar{P}_{i}>0, \bar{F}_{0}, \bar{F}_{i}, S_{0}$, $S_{i}, \Lambda_{i}, \Xi_{i j}$ and $Z_{i}$ such that

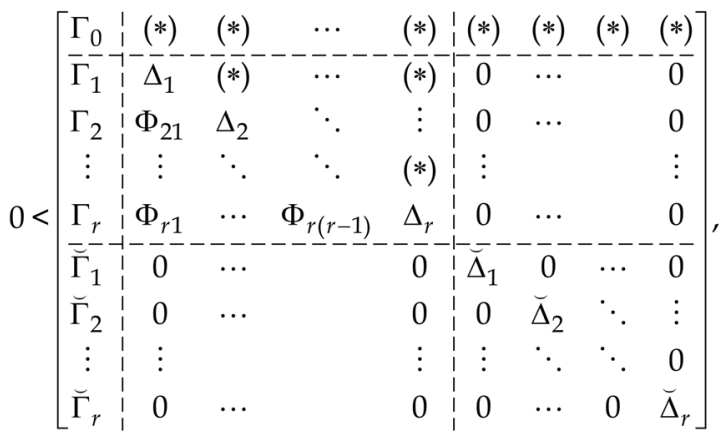

$$
\begin{aligned}
& 0 \leq \Lambda_{i}+\Lambda_{i}^{T}, 0 \leq \Xi_{i j}+\Xi_{i j}^{T}, 0 \leq Z_{i}+Z_{i}^{T}, \forall i, j \in[1, r], j \neq i .
\end{aligned}
$$

Then, the closed-loop system in (72) is asymptotically stable for all admissible grades satisfying the constraints (46). Moreover, the controller is given by

$$
F(\theta(t))=\left(\bar{F}_{0}+\sum_{i=1}^{r} \theta_{i}(t) \bar{F}_{i}\right)\left(\bar{P}+\sum_{i=1}^{r} \theta_{i}(t) \bar{P}_{i}\right)^{-1} .
$$

Proof The condition in (87) is equivalent to

$$
\begin{aligned}
0<\mathcal{L}(\theta(t)) \triangleq & \dot{\bar{P}}(\theta(t))-\bar{P}(\theta(t)) A^{T}(\theta(t))-\bar{F}^{T}(\theta(t)) B^{T}(\theta(t)) \\
& -A(\theta(t)) \bar{P}(\theta(t))-B(\theta(t)) \bar{F}(\theta(t))
\end{aligned}
$$

which can be rewritten by (70), (75), (89) into

$$
\begin{aligned}
0 & <\mathcal{L}_{0}+\sum_{i=1}^{r} \theta_{i}(t)\left(\mathcal{L}_{i}+\mathcal{L}_{i}^{T}\right)+\sum_{i=1}^{r} \dot{\theta}_{i}(t)\left(\breve{\mathcal{L}}_{i}+\breve{\mathcal{L}}_{i}^{T}\right)+\sum_{i=1}^{r} \theta_{i}^{2}(t) \mathcal{L}_{i i} \\
& +\sum_{i=1}^{r}\left(\sum_{j=1}^{i-1} \theta_{i}(t) \theta_{j}(t) \mathcal{L}_{i j}+\sum_{j=i+1}^{r} \theta_{i}(t) \theta_{j}(t) \mathcal{L}_{i j}^{T}\right)
\end{aligned}
$$

where

$$
\mathcal{L}_{0}=-\left(A_{0} \bar{P}+B_{0} \bar{F}_{0}+\bar{P} A_{0}^{T}+\bar{F}_{0}^{T} B_{0}^{T}\right),
$$




$$
\begin{gathered}
\mathcal{L}_{i}=-\left(A_{0} \bar{P}_{i}+A_{i} \bar{P}+B_{0} \bar{F}_{i}+B_{i} \bar{F}_{0}\right), \overline{\mathcal{L}}_{i}=\frac{1}{2} \bar{P}_{i}, \\
\mathcal{L}_{i i}=-\left(A_{i} \bar{P}_{i}+B_{i} \bar{F}_{i}+\bar{P}_{i} A_{i}^{T}+\bar{F}_{i}^{T} B_{i}^{T}\right), \\
\mathcal{L}_{i j}=-\left(A_{i} \bar{P}_{j}+B_{i} \bar{F}_{j}+A_{j} \bar{P}_{i}+B_{j} \bar{F}_{i}\right) .
\end{gathered}
$$

Hence, reminding the relaxation technique in Case 2, we can clearly see that the condition $\mathcal{L}(\theta(t))>0$ subject to (46) is guaranteed by (90) and (91), where

$$
\begin{gathered}
\Gamma_{0}=\mathcal{L}_{0}-S_{0}-S_{0}^{T}+\sum_{i=1}^{r} \rho_{i} v_{i}\left(Z_{i}+Z_{i}^{T}\right), \Gamma_{i}=\mathcal{L}_{i}-\beta_{i} \Lambda_{i}+S_{0}-S_{i}, \\
\breve{\Gamma}_{i}=\breve{\mathcal{L}}_{i}-\left(\rho_{i}+v_{i}\right) Z_{i}, \Delta_{i}=\mathcal{L}_{i i}+\left(\Lambda_{i}+\Lambda_{i}^{T}\right)+\left(S_{i}+S_{i}^{T}\right), \\
\breve{\Delta}_{i}=Z_{i}+Z_{i}^{T}, \Phi_{i j}=\mathcal{L}_{i j}+\left(S_{i}+S_{j}\right)-\left(\Xi_{i j}+\Xi_{j i}\right) .
\end{gathered}
$$

\section{Stabilization of discrete-time LPV systems}

Consider the following discrete-time T-S fuzzy systems:

$$
x(k+1)=A(\theta(k)) x(k)+B(\theta(k)) u(k),
$$

where $x(k) \in R^{n_{x}}$ and $u(k) \in R^{n_{u}}$ denote the state and control input, respectively; $\theta(k)$ denotes the time-varying parameter vector; and

$$
A(\theta(k))=\sum_{i=1}^{r} \theta_{i}(k) A_{i}, B(\theta(k))=\sum_{i=1}^{r} \theta_{i}(k) B_{i} .
$$

For the stabilization of (101), we shall consider a state-feedback controller dependent not only the current-time parameter vector $\theta(k)$ but also on the one-step-past vector $\theta(k-1)$ for time $k$ :

$$
u(k)=F(\theta(k-1), \theta(k)) x(k) .
$$

Remark 3: The reason for using both $\theta(k)$ and $\theta(k-1)$ in (103) is twofold. One is to enhance the causality between the control gain and the Lyapunov function whose forward difference is a function of $\theta(k)$ and $\theta(k-1)$. The other is to use the information existing between $\theta(k-1)$ and $\theta(k)$ as well as the instant information $\theta(k)$ when performing the control action (Choi \& Park, 2003; Kim et al, 2004).

As a result, the resulting closed-loop system under (103) is described as follows:

$$
x(k+1)=\hat{A}(\theta(k-1), \theta(k)) x(k),
$$


where

$$
\hat{A}(\theta(k-1), \theta(k)) \triangleq A(\theta(k))+B(\theta(k)) F(\theta(k-1), \theta(k)) .
$$

\section{Stabilization using CQLF:}

First, let us consider a common quadratic Lyapunov function $V(x(k))$ :

$$
V(x(k))=x^{T}(k) P x(k), P>0,
$$

whose forward difference along the closed-loop system trajectories is given by

$$
\Delta V(x(k))=V(x(k+1))-V(x(k))=x^{T}(k+1) P x(k+1)-x^{T}(k) P x(k) .
$$

Then, the stabilization condition for the closed-loop system in (104) is readily given as follows:

$$
0<P-\hat{A}^{T}(\theta(k-1), \theta(k)) P \hat{A}(\theta(k-1), \theta(k)) \text {. }
$$

Theorem 5: (PLMI-based condition) Suppose that there exist matrices $\bar{P}$ and $\bar{F}(\theta(k-1), \theta(k))$ such that

$$
0<\left[\begin{array}{cc}
\bar{P} & (*) \\
A(\theta(k)) \bar{P}+B(\theta(k)) \bar{F}(\theta(k-1), \theta(k)) & \bar{P}
\end{array}\right],
$$

where $\bar{P}=P^{-1}$ and $\bar{F}(\theta(k-1), \theta(k))=F(\theta(k-1), \theta(k)) \bar{P}$. Then, the closed-loop system in (104) is asymptotically stable for all admissible grades $\theta(k-1)$ and $\theta(k)$. Moreover, the controller is given by

$$
F(\theta(k-1), \theta(k))=\bar{F}(\theta(k-1), \theta(k)) \bar{P}^{-1} .
$$

Proof The proof is straightforward, and hence is omitted here.

In the following, we shall derive a finite number of LMIs from (109). To this end, let us first take

$$
\bar{F}(\theta(k-1), \theta(k))=\sum_{i=1}^{r} \theta_{i}^{-} \bar{F}_{i}^{-}+\bar{F}(\theta(k)), \bar{F}(\theta(k))=\sum_{i=1}^{r} \theta_{i} \bar{F}_{i} .
$$

Then, we can obtain the following theorem with the help of the relaxation scheme in Case 1. Theorem 6: (LMI-based condition) Suppose that there exist matrices $\bar{P}>0, \bar{F}_{i}^{-}, \bar{F}_{i}, S_{0}, S_{i}, \Lambda_{i}$ and $\Xi_{i j}$ such that

$$
0<\left[\begin{array}{c:cccc}
\Gamma_{0} & (*) & (*) & \cdots & (*) \\
\hdashline \Gamma_{1}^{(l)} & \Delta_{1} & (*) & \cdots & (*) \\
\Gamma_{2}^{(l)} & \Phi_{21} & \Delta_{2} & \ddots & \vdots \\
\vdots & \vdots & \ddots & \ddots & (*) \\
\Gamma_{r}^{(l)} & \Phi_{r 1} & \cdots & \Phi_{r(r-1)} & \Delta_{r}
\end{array}\right], \forall l \in[1, r]
$$




$$
0 \leq \Lambda_{i}+\Lambda_{i}^{T}, 0 \leq \Xi_{i j}+\Xi_{j i}^{T}, \forall i, j \in[1, r], j \neq i
$$

Then, the closed-loop system in (104) is asymptotically stable for all admissible grades $\theta(k-1)$ and $\theta(k)$. Moreover, the controller is given by

$$
F(\theta(k-1), \theta(k))=\left(\sum_{i=1}^{r} \theta_{i}^{-} \bar{F}_{i}^{-}+\sum_{i=1}^{r} \theta_{i} \bar{F}_{i}\right)^{-1} \bar{P}^{-1}
$$

Proof Define

$$
\mathcal{L}_{l}(\theta(k)) \triangleq\left[\begin{array}{cc}
\bar{P} & \left.{ }^{*}\right) \\
A(\theta(k)) \bar{P}+B(\theta(k))\left(\bar{F}_{l}^{-}+\bar{F}(\theta(k))\right) & \bar{P}
\end{array}\right] .
$$

Then, the PLMI-based condition in (109) can be written as follows:

$$
0<\sum_{l=1}^{r} \theta_{l}^{-} \mathcal{L}_{l}(\theta(k))
$$

which is equivalent to $0<\mathcal{L}_{l}(\theta(k))$, for all $l \in[1, r]$, that is,

$$
0<\mathcal{L}_{0}+\sum_{i=1}^{r} \theta_{i}(k)\left(\mathcal{L}_{i}^{(l)}+\mathcal{L}_{i}^{(l) T}\right)+\sum_{i=1}^{r} \theta_{i}^{2}(k) \mathcal{L}_{i i}+\sum_{i=1}^{r}\left(\sum_{j=1}^{i-1} \theta_{i}(k) \theta_{j}(k) \mathcal{L}_{i j}+\sum_{j=i+1}^{r} \theta_{i}(k) \theta_{j}(k) \mathcal{L}_{i j}^{T}\right)
$$

where

$$
\begin{gathered}
\mathcal{L}_{0}=\left[\begin{array}{cc}
\bar{P} & 0 \\
0 & \bar{P}
\end{array}\right], \mathcal{L}_{i}^{(l)}=\left[\begin{array}{cc}
0 & 0 \\
A_{i} \bar{P}+B_{i} \bar{F}_{l}^{-} & 0
\end{array}\right], \\
\mathcal{L}_{i i}=\left[\begin{array}{cc}
0 & (*) \\
B_{i} \bar{F}_{i} & 0
\end{array}\right], \mathcal{L}_{i j}=\left[\begin{array}{cc}
0 & 0 \\
B_{i} \bar{F}_{j}+B_{j} \bar{F}_{i} & 0
\end{array}\right] .
\end{gathered}
$$

Hence, reminding the relaxation technique in Case 1, we can clearly see that the condition $0<\mathcal{L}_{l}(\theta(k))$ subject to (34) is guaranteed by (112) and (113), where

$$
\begin{gathered}
\Gamma_{0}=\mathcal{L}_{0}-S_{0}-S_{0}^{T}, \Gamma_{i}^{(l)}=\mathcal{L}_{i}^{(l)}-\beta_{i} \Lambda_{i}+S_{0}-S_{i}, \\
\Delta_{i}=\mathcal{L}_{i i}+\left(\Lambda_{i}+\Lambda_{i}^{T}\right)+\left(S_{i}+S_{i}^{T}\right), \\
\Phi_{i j}=\mathcal{L}_{i j}+\left(S_{i}+S_{j}\right)-\left(\Xi_{i j}+\Xi_{j i}\right) .
\end{gathered}
$$

\section{Stabilization using FWLF-APD:}

Consider an FWLF $V(x(k))$ of the following form: 


$$
V(x(k))=x^{T}(k) P(\theta(k)) x(k), P(\theta(k))>0,
$$

whose forward difference along the closed-loop system trajectories is given by

$$
\Delta V(x(k))=x^{T}(k+1) P(\theta(k+1)) x(k+1)-x^{T}(k) P(\theta(k)) x(k) .
$$

Then, the stabilization condition for (104) is readily given as follows:

$$
0<P(\theta(k))-\hat{A}^{T}(\theta(k-1), \theta(k)) P(\theta(k+1)) \hat{A}(\theta(k-1), \theta(k)) .
$$

Theorem 7: (PLMI-based condition) Suppose that there exist matrices $\bar{P}(\theta(k+1)), \bar{P}(\theta(k))$, and $\bar{F}(\theta(k-1), \theta(k))$ such that

$$
0<\left[\begin{array}{cc}
\bar{P}(\theta(k)) & (*) \\
A(\theta(k)) \bar{P}(\theta(k))+B(\theta(k)) \bar{F}(\theta(k-1), \theta(k)) & \bar{P}(\theta(k+1))
\end{array}\right],
$$

where $\bar{P}(\cdot)=P^{-1}(\cdot)$ and $\bar{F}(\theta(k-1), \theta(k))=F(\theta(k-1), \theta(k)) \bar{P}(\theta(k))$. Then, the closed-loop system in (104) is asymptotically stable for all admissible grades. Moreover, the controller is reconstructed as follows:

$$
F(\theta(k-1), \theta(k))=\bar{F}(\theta(k-1), \theta(k)) \bar{P}^{-1}(\theta(k)) .
$$

Proof The proof is straightforward, and hence is omitted here.

In the following, we shall derive a finite number of LMIs from (126). To this end, let us first take

$$
\begin{gathered}
\bar{P}(\theta(k))=\bar{P}+\sum_{i=1}^{r} \theta_{i}(k) \bar{P}, \bar{P}>0, \bar{P}_{i}>0, \\
\bar{F}(\theta(k-1), \theta(k))=\sum_{i=1}^{r} \theta_{i}(k-1) \bar{F}_{i}^{-}+\bar{F}(\theta(k)), \bar{F}(\theta(k))=\sum_{i=1}^{r} \theta_{i}(k) \bar{F}_{i} .
\end{gathered}
$$

Then, we can obtain the following theorem with the help of the relaxation technique in Case 1. Theorem 8: (LMI-based condition) Suppose that there exist matrices $\bar{P}>0, \bar{P}_{i}>0, \bar{F}_{i}^{-}, \bar{F}_{i}, S_{0}$, $S_{i}, \Lambda_{i}$, and $\Xi_{i j}$ such that

$$
\begin{gathered}
0<\left[\begin{array}{c:cccc}
\Gamma_{0}^{(s)} & (*) & (*) & \cdots & (*) \\
\hdashline \Gamma_{1}^{(l)} & \Delta_{1} & (*) & \cdots & (*) \\
\Gamma_{2}^{(l)} & \Phi_{21} & \Delta_{2} & \ddots & \vdots \\
\vdots & \vdots & \ddots & \ddots & (*) \\
\Gamma_{r}^{(l)} & \Phi_{r 1} & \cdots & \Phi_{r(r-1)} & \Delta_{r}
\end{array}\right], \forall l, s \in[1, r], \\
0 \leq \Lambda_{i}+\Lambda_{i}^{T}, 0 \leq \Xi_{i j}+\Xi_{j i}^{T}, \forall i, j \in[1, r], j \neq i .
\end{gathered}
$$


Then, the closed-loop system in (104) is asymptotically stable for all admissible grades satisfying the constraints (34). Moreover, the controller is given by

$$
F(\theta(k-1), \theta(k))=\left(\sum_{i=1}^{r} \theta_{i}(k-1) \bar{F}_{i}^{-}+\sum_{i=1}^{r} \theta_{i}(k) \bar{F}_{i}\right)\left(\bar{P}+\sum_{i=1}^{r} \theta_{i}(k) \bar{P}_{i}\right)^{-1}
$$

Proof Define

$$
\mathcal{L}_{l s}(\theta(k)) \triangleq\left[\begin{array}{cc}
\bar{P}(\theta(k)) & (*) \\
A(\theta(k)) \bar{P}(\theta(k))+B(\theta(k))\left(\bar{F}_{l}^{-}+\bar{F}(\theta(k))\right) & \bar{P}+\bar{P}_{s}
\end{array}\right] .
$$

Then, the PLMI condition (126) can be rewritten as follows:

$$
0<\sum_{l=1 s=1}^{r} \sum_{s}^{r} \theta_{l}(k-1) \theta_{s}(k+1) \mathcal{L}_{l s}(\theta(k))
$$

which is equivalent to $0<\mathcal{L}_{l s}(\theta(k))$, for all $l, s \in[1, r]$, that is,

$$
0<\mathcal{L}_{0}^{(s)}+\sum_{i=1}^{r} \theta_{i}(k)\left(\mathcal{L}_{i}^{(l)}+\mathcal{L}_{i}^{(l) T}\right)+\sum_{i=1}^{r} \theta_{i}^{2}(k) \mathcal{L}_{i i}+\sum_{i=1}^{r}\left(\sum_{j=1}^{i-1} \theta_{i}(k) \theta_{j}(k) \mathcal{L}_{i j}+\sum_{j=i+1}^{r} \theta_{i}(k) \theta_{j}(k) \mathcal{L}_{i j}^{T}\right)
$$

where

$$
\begin{gathered}
\mathcal{L}_{0}^{(s)}=\left[\begin{array}{cc}
\bar{P} & 0 \\
0 & \bar{P}+\bar{P}_{s}
\end{array}\right], \mathcal{L}_{i}^{(l)}=\left[\begin{array}{cc}
\frac{1}{2} \bar{P}_{i} & 0 \\
A_{i} \bar{P}+B_{i} \bar{F}_{l}^{-} & 0
\end{array}\right], \\
\mathcal{L}_{i i}=\left[\begin{array}{cc}
0 & (*) \\
A_{i} \bar{P}_{i}+B_{i} \bar{F}_{i} & 0
\end{array}\right], \mathcal{L}_{i j}=\left[\begin{array}{cc}
0 & 0 \\
A_{i} \bar{P}_{j}+A_{j} \bar{P}_{i}+B_{i} \bar{F}_{j}+B_{j} \bar{F}_{i} & 0
\end{array}\right] .
\end{gathered}
$$

Hence, reminding the relaxation technique in Case 1, we can clearly see that the condition $0<\mathcal{L}_{l s}(\theta(k))$ subject to (34) is guaranteed by (130) and (131), where

$$
\begin{gathered}
\Gamma_{0}^{(s)}=\mathcal{L}_{0}^{(s)}-S_{0}-S_{0}^{T}, \Gamma_{i}^{(l)}=\mathcal{L}_{i}^{(l)}-\beta_{i} \Lambda_{i}+S_{0}-S_{i}, \\
\Delta_{i}=\mathcal{L}_{i i}+\left(\Lambda_{i}+\Lambda_{i}^{T}\right)+\left(S_{i}+S_{i}^{T}\right), \\
\Phi_{i j}=\mathcal{L}_{i j}+\left(S_{i}+S_{j}\right)-\left(\Xi_{i j}+\Xi_{j i}\right) .
\end{gathered}
$$

\section{Stabilization using FWLF-QPD:}

The relevant formulation has been given in our previous research works (Kim \& Park, 2008), associate with the $\mathcal{H}_{\infty}$ performance. In the derivation, the $\mathcal{H}_{\infty}$ stabilization conditions are 
formulated in terms of PLMIs, which are reconverted into a finite set of LMI-based conditions with the help of the relaxation technique in Case 3.

\section{Acknowledgment}

This research was supported by WCU (World Class University) program through the Korea Science and Engineering Foundation funded by the Ministry of Education, Science and Technology (Project No. R31-2008-000-10100-0).

\section{References}

[1] M. Sugeno, "On stability of fuzzy systems expressed by fuzzy rules with singleton consequents," IEEE Trans. Fuzzy Syst., vol. 7, no. 2, pp. 201-224, 1999.

[2] T. Takagi and M. Sugeno, "Fuzzy identification of systems and its applications to modeling and control," IEEE Trans. Syst., Man, Cybern., vol. SMC-15, no. 1, pp. 116$-132,1985$.

[3] K. Tanaka and M. Sugeno, "Stability analysis and design of fuzzy control systems," Fuzzy Sets Syst., vol. 45, pp. 135-156, 1992.

[4] K. Tanaka, T. Ikeda, and H. O. Wang, "Robust stabilization of a class of uncertain nonlinear systems via fuzzy control: Quadratic stabilizability $\mathcal{H}_{\infty}$ control theory, and linear matrix inequality," IEEE Trans. Fuzzy Syst., vol. 4, no. 1, pp. 1-13, 1996.

[5] H. O.Wang, K. Tanaka, and M. Griffin, "An approach to fuzzy control on nonlinear sytems: Stability and design issues," IEEE Trans. Fuzzy Syst., vol. 4, no. 1, pp. 1423, Feb. 1996.

[6] Y.-Y. Cao and P. M. Frank, "Robust $\mathcal{H}_{\infty}$ disturbance attenuation for a class of uncertain discrete-time fuzzy systems," IEEE Trans. Fuzzy Syst., vol. 8, no. 4, pp. 406-415, 2000.

[7] W. Assawinchaichote, S. K. Nguang, and P. Shi, " $\mathcal{H}_{\infty}$ output feedback control design for uncertain fuzzy singularly perturbed systems: An LMI approach," Automatica, vol. 40, no. 12, pp. 2147-2152, Dec. 2004.

[8] S.-G. Cao, N. W. Ress, and G. Feng, "Stability analysis and design for a class of continuous time fuzzy control system," Int. J. Control, vol. 64, pp. 1069-1087, 1996.

[9] S.-G. Cao, N. W. Ress, and G. Feng, "Analysis and design for a class of complex control systems, Part II: Fuzzy controller design," Automatica, vol. 33, pp. 1029-1039, 1997.

[10] S.-G. Cao, N. W. Ress, and G. Feng, "Analysis and design of fuzzy control systems using dynamic fuzzy-state space models," IEEE Trans. Fuzzy Syst., vol. 7, no. 2, pp. 192-200, Apr. 1999.

[11] Z. X. Han, G. Feng, B. L. Walcott, and J. Ma, “Dynamic output feedback controller design for fuzzy systems," IEEE Trans. Syst., Man, Cybern., B, Cybern., vol. 30, no. 1, pp. 204-210, Feb. 2000.

[12] G. Feng, "Controller synthesis of fuzzy dynamic systems based on piecewise Lyapunov functions," IEEE Trans. Fuzzy Syst., vol. 11, no. 10, pp. 605-612, Oct. 2003.

[13] G. Feng, " $\mathcal{H}_{\infty}$ controller design of fuzzy dynamic systems based on piecewise Lyapunov functions," IEEE Trans. Syst., Man, Cybern., B., Cybern., vol. 34, no. 1, pp. 283-292, Feb. 2004.

[14] C. L. Chen, G. Feng, and X. P. Guan, "Delay-dependent stability analysis and controller synthesis for discrete time T-S fuzzy systems with time delays," IEEE Trans. Fuzzy Syst., vol. 13, no. 5, pp. 630-643, Oct. 2005. 
[15] K. Tanaka, T.Hori, and H.O.Wang, "A fuzzy Lyapunov approach to fuzzy conrol system design," in Proc. Amer. Control. Conf., 2001, pp. 4790-4795.

[16] P. Park and D. J. Choi, "LPV controller design for the nonlinear RTAC system," Int. J. Robust Nonlinear Control, vol. 11, pp. 1342-1363, 2001.

[17] D. J. Choi and P. Park, " $\mathcal{H}_{\infty}$ state-feedback controller design for discrete-time fuzzy systems using fuzzy weighting-dependent Lyapunov functions," IEEE Trans. Fuzzy Syst., vol. 11, no. 2, pp. 271-278, 2003.

[18] S. H. Kim and P. Park, " $\mathcal{H}_{\infty}$ state-feedback control design for fuzzy systems using Lyapunov functions with quadratic dependence on fuzzy weighting functions," IEEE Trans. Fuzzy Syst., vol. 16, no. 6, pp. 1655-1663, 2008.

[19] S. H. Kim, C. H. Lee, and P. Park, " $\mathcal{H}_{\infty}$ state-feedback control for fuzzy systems with input saturation via fuzzy weighting-dependent Lyapunov functions," Computers $\mathcal{E}$ Mathmatics with applications, vol. 57, no. 6, pp. 981-990, 2009.

[20] B. S. Chen, C. S. Tseng, and H. J. Uang, “Robustness design of nonlinear dynamic systems via fuzzy linear control," IEEE Trans. Fuzzy Syst., vol. 7, no. 5, pp. 571-585, Oct. 1999.

[21] S.-H. Tsai and T.-H. S. Li, "Robust fuzzy control of a class of fuzzy bilinear systems with time-delay," Chaos, Solitons and Fractals, vol. 39, pp. 2028-2040, 2009.

[22] B. S. Chen, C. S. Tseng, and H. J. Uang, "Mixed $\mathcal{H}_{2} / \mathcal{H}_{\infty}$ fuzzy output feedback control design for nonlinear dynamic systems: An LMI approach," IEEE Trans. Fuzzy Syst., vol. 8, no. 3, pp. 249-265, Jun. 2000.

[23] S. Zhou, G. Feng, J. Lam, and S. Xu, "Robust $\mathcal{H}_{\infty}$ control for discrete-time fuzzy systems via basis-dependent Lyapunov functions," Information Sciences, vol. 174, pp. 197-217, 2005.

[24] L. Xiaodong and Z. Qingling, "New approaches to $\mathcal{H}_{\infty}$ controller designs based on fuzzy observers for T-S fuzzy systems via LMI," Automatica, vol. 39, pp. 1571-1582, 2003.

[25] C.-L. Hwang and L.-J. Chang, "Internet-based smart-space navigation of a car-like wheeled robot using fuzzy-neural adaptive control," IEEE Trans. Fuzzy Syst., vol. 16, no. 5, pp. 1271-1284, Oct. 2008.

[26] X. Jiang and Q.-L. Han, “On designing fuzzy controllers for a class of nonlinear networked control systems," IEEE Trans. Fuzzy Syst., vol. 16, no. 4, pp. 1050-1060, Aug. 2008.

[27] H. Gao, Y. Zhao, and T. Chen, " $\mathcal{H}_{\infty}$ fuzzy control of nonlinear systems under unreliable communication links," IEEE Trans. Fuzzy Syst., vol. 17, no. 2, pp. 265278, Apr. 2009.

[28] Y.-Y. Cao and P. M. Frank, "Analysis and synthesis of nonlinear time-delay systems via fuzzy control approach," IEEE Trans. Fuzzy Syst., vol. 8, no. 2, pp. 200-211, Apr. 2000.

[29] C.-L. Chen, G. Feng, and X.-P. Guan, "Delay-dependent stability analysis and controller synthesis for discrete-time T-S fuzzy systems with time delays," IEEE Trans. Fuzzy Syst., vol. 13, no. 5, pp. 630-643, Oct. 2005.

[30] H.-N. Wu, "Delay-dependent $\mathcal{H}_{\infty}$ fuzzy observer-based control for discrete-time nonlinear systems with state delay," Fuzzy Sets and Systems, vol. 159, pp. 2696-2712, 2008.

[31] G. Feng, "A survey on analysis and design of model-based fuzzy control systems," IEEE Trans. Fuzzy Syst., vol. 14, no. 5, pp. 676-697, 2006. 
[32] G. Becker, A. Packard, D. Philbrick, and G. Balas, “Control of parametrically dependent linear systems: a single quadratic lyapunov approach," Proc. ACC-93, San Francisco, pp. 2795-2799, 1993.

[33] T. Hu and Z. Lin, "Control systems with actuator saturation: Analysis and design," Vol. xvi (392p). Boston: Birkhäuser.

[34] Q. Zhao and J. Jiang, "Reliable state feedback control system design against actuator failures," Automatica, vol. 34(10), pp. 1267-1272, 1998.

[35] G. H. Yang, J. L. Wang, and Y. C. Soh, "Reliable $\mathcal{H}_{\infty}$ controller design for linear systems," Automatica, vol. 37(5), pp. 717-725, 2001.

[36] F. Liao, J. L. Wang, and G. H. Yang, "Reliable robust flight tracking control: An LMI approach," IEEE Trans. Control Syst. Technol., vol. 10(1), pp. 76-89, 2002.

[37] H. N. Wu and H. Y. Zhang, "Reliable $\mathcal{H}_{\infty}$ fuzzy control for continuous-time nonlinear systems with actuator failures," IEEE Trans. Fuzzy Syst., vol. 14(5), pp. 600-618, 2006.

[38] Y. Nesterov and A. Nemirovski, "Interior point polynomial methods in convex programming theory and applicqations," Philadelpia, PA, SIAM, 1994.

[39] S. Boyd, L. E. Chauoi, E. Feron, and V. Balakrishan, Linear Matrix Inequalities in System and Control Theory. Philadelphia, PA:SIAM, 1994.

[40] L. E. Gahoui, F. Oustry, and H. Levert, "Robust solutions to uncertain semidefinite programs," SIAM Journal of Control and Optimization, vol. 9(1), pp. 33-52, 1998.

[41] K. Tanaka, T. Ikeda, and H. O. Wang, "Fuzzy regulators and fuzzy observers: relaxed stability conditins and LMI-based designs," IEEE Trans. Fuzzy Syst., vol. 6(2), pp. 250-265, 1998.

[42] E. Kim and H. Lee, "New approaches to relaxed quadratic stablity conditin of fuzzy control systems," IEEE Trans. Fuzzy Syst., vol. 8(5), pp. 523-534, 2000.

[43] L. Xiaodong and Z. Qingling, "New approaches to $\mathcal{H}_{\infty}$ controller designs based on fuzzy observers for T-S fuzzy systems via LMI," Automatica, vol. 39, pp. 1571-1582, 2003.

[44] H. D. Tuan, P. Apkarian, T. Narikiyo, and Y. Yamamoto, "Parameterized linear matrix inequality techniques in fuzzy control system design," IEEE Trans. Fuzzy Syst., vol. 9(2), pp. 324-332, 2001.

[45] M.C.M. Teixeira, E. Assuncão, and R.G. Avellar, "On relaxed LMI-based designs for fuzzy regulators and fuzzy observers," IEEE Trans. Fuzzy Syst., vol.11 pp.613-623, 2003.

[46] A. Sala and C. Ariño, "Relaxed stability and performance conditions for TakagiSugeno fuzzy systems with knowledge on membership function overlap," IEEE Trans. Syst., Man., Cybern. B, Cybern., vol. 37(3), pp. 727-732, 2007.

[47] C.-H. Fang, Y.-S. Liu, S.-W. Kau, L. Hong, and C.-H. Lee, “A new LMI-based approach to relaxed quadratic stabilization of T-S fuzzy control systems," IEEE Trasn. Fuzzy Syst., vol. 14(3), pp. 386-397, 2006.

[48] M. C. de Oliveira, R. E. Skelton, Stability tests for constrained linear systems, in Perspectives in Robust Control, S. O. Reza Moheimani, Editor. New York: Springer-Verlag, 2001, vol. 268, series Lecture Notes in Control and Information Sciences, pp. 241-257.

[49] L. Fang, H. Lin, and P. J. Antsaklis, "Stabilization and performance analysis for a class of switched systems," in Proceedings of the 43rd IEEE Conference on Decision and Control, Atlantis, Paradise Island, Bahamas, Dec. 2004, pp. 3265-3270.

[50] D. K. Kim, P.-G. Park and J. W. Ko, "Output-feedback $\mathcal{H}_{\infty}$ control of systems over communication networks using a deterministic switching system approach," Automatica, 40, pp. 1205-1212, 2004. 


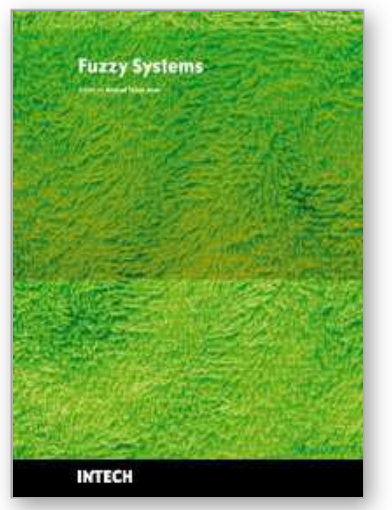

\author{
Fuzzy Systems \\ Edited by Ahmad Taher Azar
}

ISBN 978-953-7619-92-3

Hard cover, 216 pages

Publisher InTech

Published online 01, February, 2010

Published in print edition February, 2010

While several books are available today that address the mathematical and philosophical foundations of fuzzy logic, none, unfortunately, provides the practicing knowledge engineer, system analyst, and project manager with specific, practical information about fuzzy system modeling. Those few books that include applications and case studies concentrate almost exclusively on engineering problems: pendulum balancing, truck backeruppers, cement kilns, antilock braking systems, image pattern recognition, and digital signal processing. Yet the application of fuzzy logic to engineering problems represents only a fraction of its real potential. As a method of encoding and using human knowledge in a form that is very close to the way experts think about difficult, complex problems, fuzzy systems provide the facilities necessary to break through the computational bottlenecks associated with traditional decision support and expert systems. Additionally, fuzzy systems provide a rich and robust method of building systems that include multiple conflicting, cooperating, and collaborating experts (a capability that generally eludes not only symbolic expert system users but analysts who have turned to such related technologies as neural networks and genetic algorithms). Yet the application of fuzzy logic in the areas of decision support, medical systems, database analysis and mining has been largely ignored by both the commercial vendors of decision support products and the knowledge engineers who use them.

\title{
How to reference
}

In order to correctly reference this scholarly work, feel free to copy and paste the following:

Sung Hyun Kim and PooGyeon Park (2010). Control of T-S Fuzzy Systems Using Fuzzy Weighting-Dependent Lyapunov Function, Fuzzy Systems, Ahmad Taher Azar (Ed.), ISBN: 978-953-7619-92-3, InTech, Available from: http://www.intechopen.com/books/fuzzy-systems/control-of-t-s-fuzzy-systems-using-fuzzy-weightingdependent-lyapunov-function

\section{INTECH}

open science | open minds

\section{InTech Europe}

University Campus STeP Ri

Slavka Krautzeka 83/A

51000 Rijeka, Croatia

Phone: +385 (51) 770447

Fax: +385 (51) 686166

\section{InTech China}

Unit 405, Office Block, Hotel Equatorial Shanghai

No.65, Yan An Road (West), Shanghai, 200040, China 中国上海市延安西路65号上海国际贵都大饭店办公楼405单元

Phone: +86-21-62489820

Fax: +86-21-62489821 
www.intechopen.com 
(C) 2010 The Author(s). Licensee IntechOpen. This chapter is distributed under the terms of the Creative Commons Attribution-NonCommercialShareAlike-3.0 License, which permits use, distribution and reproduction for non-commercial purposes, provided the original is properly cited and derivative works building on this content are distributed under the same license. 